\title{
Flow probe of symmetry energy in relativistic heavy-ion reactions ${ }^{\star}$
}

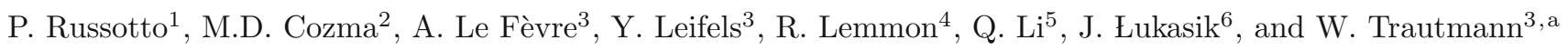 \\ 1 INFN, Sezione di Catania, I-95123 Catania, Italy \\ 2 IFIN-HH, Reactorului 30, 077125 Măgurele-Bucharest, Romania \\ 3 GSI Helmholtzzentrum für Schwerionenforschung GmbH, D-64291 Darmstadt, Germany \\ 4 STFC Daresbury Laboratory, Warrington WA4 4AD, UK \\ 5 School of Science, Huzhou Teachers College, Huzhou 313000, P.R. China \\ ${ }^{6}$ IFJ-PAN, Pl-31342 Kraków, Poland
}

Received: 23 July 2013 / Revised: 24 September 2013

Published online: 26 February 2014

(c) The Author(s) 2013. This article is published with open access at Springerlink.com

Communicated by A. Ramos

\begin{abstract}
Flow observables in heavy-ion reactions at incident energies up to about $1 \mathrm{GeV}$ per nucleon have been shown to be very useful for investigating the reaction dynamics and for determining the parameters of reaction models based on transport theory. In particular, the elliptic flow in collisions of neutron-rich heavy-ion systems emerges as an observable sensitive to the strength of the symmetry energy at suprasaturation densities. The comparison of ratios or differences of neutron and proton flows or neutron and hydrogen flows with predictions of transport models favors an approximately linear density dependence, consistent with ab initio nuclear-matter theories. Extensive parameter searches have shown that the model dependence is comparable to the uncertainties of existing experimental data. Comprehensive new flow data of high accuracy, partly also through providing stronger constraints on model parameters, can thus be expected to improve our knowledge of the equation of state of asymmetric nuclear matter.
\end{abstract}

\section{Introduction}

Heavy-ion reactions at relativistic energies and small impact parameters proceed through violent initial stages during which highly excited and compressed nuclear matter is temporarily produced. The study of these reactions is thus a means of gaining information on extremematter properties which recently has advanced to the teraelectron-volt regime at the Large Hadron Collider at the CERN laboratory [1-4].

The present paper is focussed on the lower end of the relativistic domain with incident energies between several hundred $\mathrm{MeV}$ up to about one $\mathrm{GeV}$ per nucleon. Here, the central densities may reach values of up to two or three times the saturation value while the dynamics is still dominated by hadronic degrees of freedom. This is illustrated in fig. 1 with results from isospin-dependent Boltzmann-Uehling-Uhlenbeck (IBUU) transport model calculations [5]. The high-density phase is short, typically

\footnotetext{
* Contribution to the Topical Issue "Nuclear Symmetry Energy" edited by Bao-An Li, Àngels Ramos, Giuseppe Verde, Isaac Vidaña.

a e-mail: w.trautmann@gsi.de
}

$20 \mathrm{fm} / c$, but involves up to three quarters of the total baryon multiplicity of the system (fig. 1, middle row of panels). Nuclear matter at densities of this order is believed to be present in neutron stars and also temporarily formed during the core-collapse phase of supernova explosions. Modeling these astrophysical phenomena requires the knowledge of nuclear matter properties far away from saturation and far away from symmetry. The equation of state (EoS) of neutron-rich asymmetric matter has, therefore, received particular attention recently, motivated by the impressive progress made in astrophysical observations and measurements [6-9], and especially also at high density where its behavior is least well known $[7,8,10]$.

Colliding heavy ions at relativistic energies represents a possible means for studying the nuclear equation of state at supra-saturation densities in laboratory experiments. It still remains a challenge, however, to find observables suitable for extracting information on its properties during the brief compression phase $[11,12]$. This has been an intense field of research during the past decades which has rapidly concentrated on collective flows and meson production. The pressure gradients due to compression and the increased collision rates at high density are expected to affect these observables. Studies of 


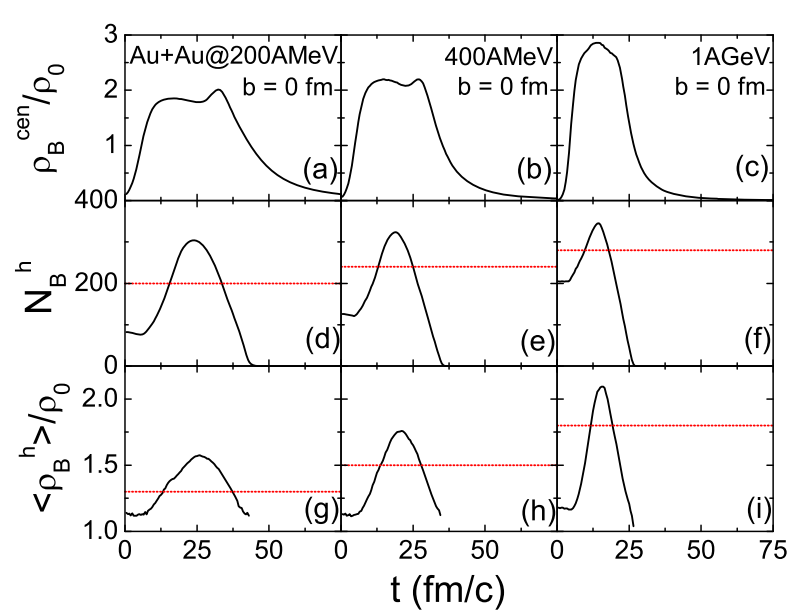

Fig. 1. Temporal evolution of central baryon density (top), baryon multiplicity (middle), and average baryon density (bottom) in the high-density $\left(\rho>\rho_{0}\right)$ phase of central ${ }^{197} \mathrm{Au}+$ ${ }^{197} \mathrm{Au}$ collisions at three energies ( $\rho_{0}$ is the saturation density). Horizontal lines indicate the average values used in the thermal model of ref. [5] (reprinted with permission from ref. [5]; Copyright (2013) by the American Physical Society).

flow and kaon production within the framework of transport theory have indeed both been essential for reaching the present consensus that a soft EoS with compressibility $K \approx 230 \mathrm{MeV}$ and momentum-dependent interactions best describes the response of symmetric nuclear matter to compression [12-15].

The same observables appear naturally as primary candidates for investigating the equation of state of asymmetric matter, the so-called asy-EoS. It is usually expressed in the form of the symmetry energy, the subject of this topical issue, which is the difference between the energies per nucleon of neutron matter and of symmetric matter. It represents the response of nuclear matter to asymmetry. Its study, consequently, requires differential observables measuring differences as a function of asymmetry or isotopic pairs of observables that respond differently to a compression of neutron-rich matter. As the symmetry energy appears in nearly every aspect of nuclear structure and reactions, a wide variety of possibilities exists. Many different kinds of constraints have been identified and quantified for the density regime below saturation, down to extremely low densities. The accompanying articles of this topical issue present a detailed picture of the various studies made and of the convergence achieved in recent years (see also, e.g., refs. [16-18]).

In the density regime exceeding saturation, the symmetry energy is still largely unknown for several reasons. Phenomenological forces are well constrained near or just below saturation but lead to largely diverging results if they are extrapolated to higher densities $[10,19]$. Microscopic many-body calculations with realistic potentials face the difficulty that three-body forces and short-range correlations are not sufficiently well known at higher densities at which their importance increases [20-22]. Chiral effective field theories have no free parameters for threebody forces, when applied to systems with only neutrons,

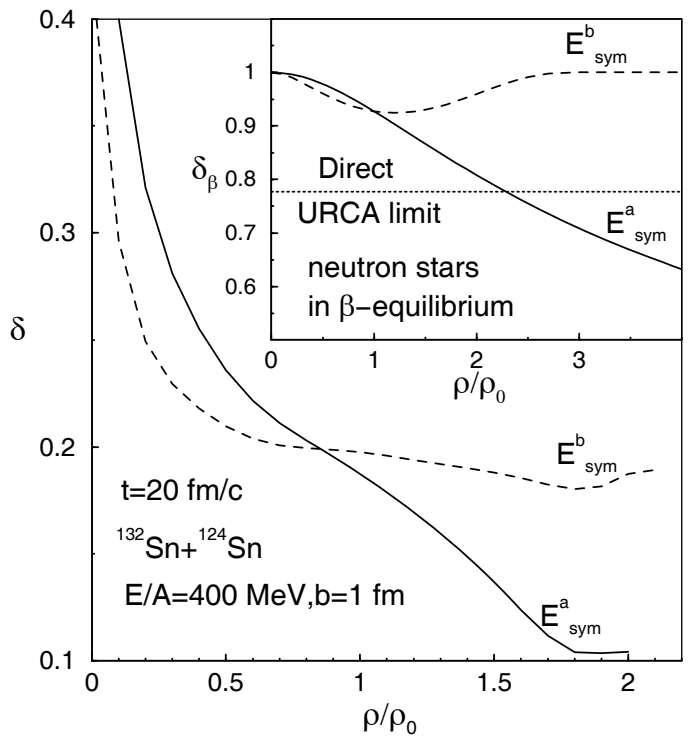

Fig. 2. Isospin asymmetry $\delta=\left(\rho_{n}-\rho_{p}\right) / \rho$ as a function of the normalized density $\rho / \rho_{0}$ at time $t=20 \mathrm{fm} / c$ in the ${ }^{132} \mathrm{Sn}+$ ${ }^{124} \mathrm{Sn}$ reaction with a stiff $\left(E_{\mathrm{sym}}^{a}\right)$ and with a soft $\left(E_{\mathrm{sym}}^{b}\right)$ density dependence of the nuclear symmetry energy. The corresponding correlation for neutron stars in $\beta$-equilibrium is shown in the inset (reprinted with permission from ref. [30]; Copyright (2002) by the American Physical Society).

but require extrapolations for reaching densities beyond saturation [23]. Even the magnitude of the kinetic contribution, related to the nuclear Fermi motion and considered as principally understood, could possibly be modified by a redistribution of nucleon momenta due to short-range correlations in high-density nuclear matter [24,25]. The need for experimental high-density probes is thus obvious, with the above-mentioned collective flows and subthreshold particle production as the main candidates.

\section{High-density probes}

Collective flows in nucleus-nucleus collisions, appearing in the form of anisotropic particle emissions as a result of collective velocity fields, have been studied rather intensely for many years (for reviews see, e.g., [26, 27]). Measurements at beam energies up to several $\mathrm{GeV}$ per nucleon were used to extract the EoS of nuclear matter from quantitative comparisons with the results of microscopic transport calculations $[11,12,28,29]$. Significant progress has been made.

The use of differential isotopic flows for the study of the asy-EoS has been suggested by Bao-An Li whose calculations with an isospin-dependent hadronic transport model indicated parallels in the density-dependent isotopic compositions of neutron stars and of the transient systems formed in collisions of neutron-rich nuclei. They exhibited a similar dependence on the high-density behavior of the nuclear symmetry energy used in the calculations [30]. This is illustrated in fig. 2 which points to the remarkable possibility of gaining information on macroscopic astrophysical objects from laboratory experiments with atomic nuclei smaller by 55 orders of magnitude in mass. 
The comparatively small asymmetry of available nuclei represents a major difficulty, however. There will be an important role to be played by radioactive secondary beams, as assumed in the example shown in fig. 2, but the symmetry effects will still be small relative to those of the dominating isoscalar forces. It is thus essential to identify differential observables as, e.g., differences or ratios of observables measured for isotopic partner systems or reactions. One hopes to enhance the response to asymmetry as the isoscalar dynamics largely cancels. The so-called differential directed flow proposed by $\mathrm{Li}[30]$ is the difference of the multiplicity-weighted directed flows of neutrons and protons. Directed flow describes the rapidity dependence of the mean in-plane transverse momenta of observed reaction products. Isotopic yield ratios or double ratios represent another possible class of observables. The double neutron-to-proton ratios obtained from isospinasymmetric but mass-symmetric pairs of reactions proposed in refs. [31,32] are being successfully used at lower energies [33].

Besides the transverse directed flow, also the elliptic flow has been studied with model calculations to test its usefulness as a probe of the asy-EoS [7,34-37]. Elliptic flow relates to the azimuthal anisotropy of particle emissions, mainly differentiating between predominantly inplane emissions as recently observed in ultrarelativistic heavy-ion collisions [1-4] and the out-of-plane emissions or squeeze-out observed in the present regime of lower energies as a consequence of the pressure build-up in the collision zone [38]. A particular encouragement was provided by transport calculations with quantum-molecular dynamics (QMD) models for ${ }^{197} \mathrm{Au}+{ }^{197} \mathrm{Au}$ collisions at $400 \mathrm{MeV}$ per nucleon according to which the elliptic flow of free neutrons and protons responds significantly differently to variations of the parameterization of the symmetry energy $[39,40]$.

A data set to test these predictions has been available from earlier experiments of the FOPI/LAND Collaboration. It was originally collected and shown to provide evidence for the squeeze-out of neutrons emitted in ${ }^{197} \mathrm{Au}+{ }^{197} \mathrm{Au}$ collisions at $400 \mathrm{MeV}$ per nucleon [41, 42]. The capability of the Large Area Neutron Detector LAND [43] used in these experiments of detecting neutrons as well as charged particles permitted the differential analysis of the observed flow patterns in the form of flow ratios [39] or flow differences [40]. Both analyses favor a density dependence between moderately soft and moderately stiff, close to the predictions of $a b$ initio calculations using realistic forces (see below) and also consistent with the observations made at sub-saturation density (see, e.g. refs. [16-18] and pertinent articles in this topical issue).

Whether meson production yields will become similarly useful for the same purpose is not so clear at present. It has been suggested that the ratio of the anti-strange kaon isospin partners, $\mathrm{K}^{+} / \mathrm{K}^{0}$, may serve as a useful observable $[44,45]$. The predicted effects were not very large but the production of kaons through $\Delta$ resonances and their weak interaction with the nuclear medium distinguish them as direct messengers from the high-density zone. Subthreshold kaon production for the isotopic pair of reactions ${ }^{96} \mathrm{Zr}+{ }^{96} \mathrm{Zr}$ and ${ }^{96} \mathrm{Ru}+{ }^{96} \mathrm{Ru}$ was studied by the FOPI Collaboration at $1.53 \mathrm{GeV}$ per nucleon [46]. The $\mathrm{K}^{+}$mesons were identified by correlating their momentum determined with the central drift chamber and their velocity measured with the time-of-flight barrel of the FOPI detector. The $\mathrm{K}_{s}^{0}$ mesons were identified via their weak decay into $\pi^{+}$and $\pi^{-}$and by reconstructing the displaced decay vertices in the central drift chamber. Because of the resulting significantly different detection efficiencies, a double ratio was formed from the $\mathrm{K}^{+} / \mathrm{K}^{0}$ production ratios obtained for the two collision systems and compared to model calculations. A significant sensitivity of this observable to the chosen stiffness of the asy-EoS was expected from the calculations for infinite nuclear matter. It diminished, however, by one order of magnitude when the calculations were performed for the actual heavy-ion collisions studied in the experiment [46]. The measured double ratio is satisfactorily reproduced irrespective of the choice made for the asy-EoS.

An even more puzzling situation is encountered in the case of the $\pi^{-} / \pi^{+}$yield ratios measured by the FOPI Collaboration at several energies up to $1.5 \mathrm{GeV}$ per nucleon and for the four mass-symmetric systems ${ }^{40} \mathrm{Ca}+{ }^{40} \mathrm{Ca}$, ${ }^{96} \mathrm{Zr}+{ }^{96} \mathrm{Zr},{ }^{96} \mathrm{Ru}+{ }^{96} \mathrm{Ru}$, and ${ }^{197} \mathrm{Au}+{ }^{197} \mathrm{Au}$ [47]. Theoretical analyses of this data set came to rather conflicting conclusions, suggesting everything from a rather stiff to a super-soft behavior of the symmetry energy [48-50]. Among them, the super-soft result has initiated a broad discussion of how it might be reconciled with observed properties of neutron stars $[48,51,52]$.

Calculations with standard parameters fell below the measured $\pi^{-} / \pi^{+}$yield ratios. Therefore, more extreme assumptions had to be made to reach the experimental values. In the analysis of Xiao et et al. [48] with the IBUU04 transport model supplemented with the Gogny-inspired momentum-dependent parameterization of the symmetry energy [53], the measured dependences on the collision system and impact parameter have been well reproduced but only by assuming a super-soft density dependence of the symmetry energy, close to the $x=+1$ case shown in fig. 3 . The analysis of Feng and Jin [49] carried out with the improved isospin-dependent QMD (ImIQMD) and a power law parameterization of the potential part of the symmetry energy yielded an equally satisfactory description of the data with the stiff choice $\gamma=2$ for the power law exponent (cf. fig. 3). Most recently, Xie et al. addressed the same issue within the Boltzmann-Langevin approach and a power law parameterization and obtained again support for a super-soft scenario for the symmetry energy [50].

This situation is clearly unfortunate because the expected variations themselves, of up to $20 \%$ for soft $v s$. stiff parameterizations, are rather large. Possible reasons for it may lie in the treatment of the $\Delta$ dynamics in transport models and in competing effects of the mean fields and $\Delta$ thresholds whose weights may be varying among the different approaches $[8,56,57]$. The role of partial cancellations of $s$-wave and $p$-wave effects in the nuclear medium which causes a reduction of the $\pi^{-} / \pi^{+}$ratios has recently been pointed out by $\mathrm{Xu}$ et al. [5]. Including the isospindependent pion in-medium effects is thus important, even 


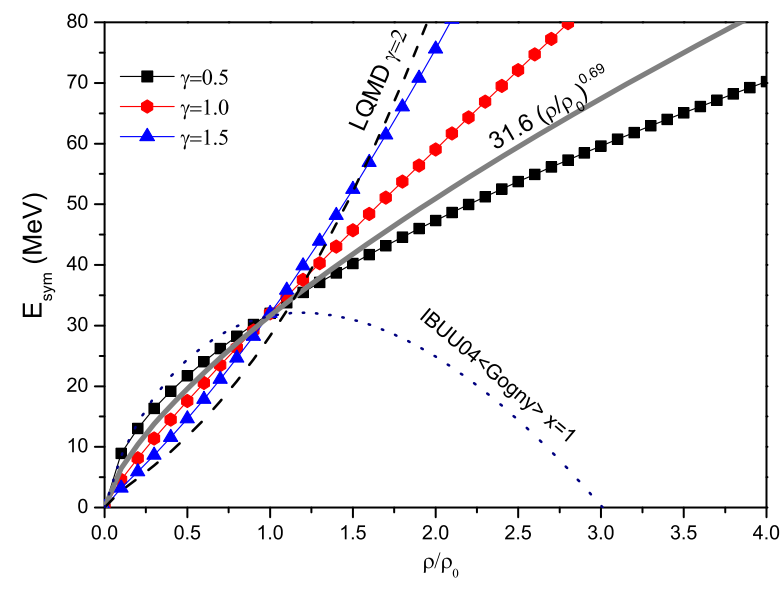

Fig. 3. Parameterizations of the nuclear symmetry energy as used in transport codes: three parameterizations of the potential term used in the UrQMD (ref. [44]) with power law coefficients $\gamma=0.5,1.0$, and 1.5 (lines with symbols as indicated), the result with $\gamma=0.69$ obtained from analyzing isospin diffusion data with the IBUU04 (full line, ref. [54]), and the super-soft and stiff parameterizations obtained from analyzing the $\pi^{-} / \pi^{+}$production ratios with the IBUU04 (dotted line, ref. [48]) and the ImIQMD (dashed line labeled LQMD, ref. [49]) transport models (from ref. [55], reprinted with kind permission from Springer Science+Business Media).

though not trivial in transport models. For a more detailed discussion and interpretation, the reader is referred to the article by Zhi-Gang Xiao et al. in this topical issue.

The conflicting interpretations of the meson data emphasize the need for reaching an improved understanding of the reaction mechanisms in order to achieve more robust results. Possible model dependences are an urgent issue also in the case of the differential elliptic flow and the conclusions regarding the density dependence of the symmetry energy obtained from there. Both interpretations of the FOPI/LAND data have addressed this question and demonstrated that the chosen differential observables are fairly stable with respect to variations of global parameters of the calculations as, e.g., the isoscalar EoS or the parameterization of the nucleon-nucleon crosssections $[39,40]$. A more comprehensive study of the effects of global parameter variations within their presently known limits has recently been completed and will be presented in the final section. It confirms the earlier results by showing that the overall model dependence is small enough to prevent it from concealing the sensitivity of the differential flows to the asy-EoS [58]. However, narrower constraints for the global parameters will be necessary if a more precise determination is to be achieved.

An urgent need to improve the statistical accuracy beyond that of the existing data set has equally become obvious from the studies performed on the FOPI/LAND data. It has initiated a dedicated measurement of collective flows in collisions of ${ }^{197} \mathrm{Au}+{ }^{197} \mathrm{Au}$ as well as of the ${ }^{96} \mathrm{Zr}+{ }^{96} \mathrm{Zr}$ and ${ }^{96} \mathrm{Ru}+{ }^{96} \mathrm{Ru}$ pair of systems carried out in 2011 at the GSI laboratory [59,60]. The LAND [43] detector has been operated together with a subset of the
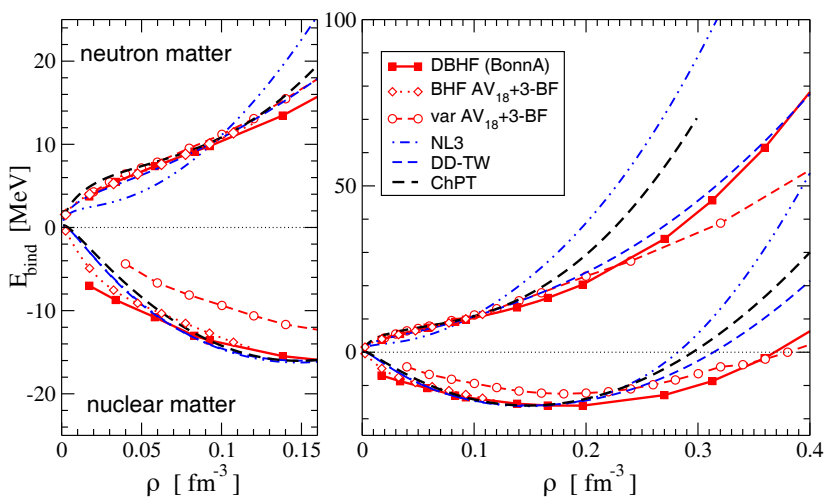

Fig. 4. EoS in nuclear matter and neutron matter. BHF/ DBHF and variational calculations with realistic forces are compared to phenomenological density functionals NL3 and DD-TW and to ChPT. The left panel zooms the low density range (from ref. [10], reprinted with kind permission from Springer Science+Business Media).

CHIMERA [61] detector array complemented with additional detector systems aiding in the measurement of the reaction plane orientation and of the flow of light fragments. Details of the experiment and the present status of the analysis will be given further below after the elliptic flow and its interpretation with transport models have been reviewed. A brief introductory overview addressing the relevance of elliptic flow in the study of the symmetry energy at supra-saturation density is available in ref. [62].

\section{Symmetry energy and parameterization}

The symmetry energy is familiar from the study of atomic masses whose dependence on their isotopic nuclear composition is accounted for with the symmetry term in the Bethe-Weizsäcker mass formula. A density dependence is already suggested by the use of individual bulk and surface terms in more refined mass formulae. Their values are, e.g., $28.1 \mathrm{MeV}$ and $33.2 \mathrm{MeV}$, respectively, in the wellknown parameterization of Myers and Swiatecki [63]. In the Fermi-gas model, the density dependence is given by a proportionality to $\left(\rho / \rho_{0}\right)^{\gamma}$ with an exponent $\gamma=2 / 3$, where $\rho_{0} \approx 0.16$ nucleons $/ \mathrm{fm}^{3}$ is the saturation density. The coefficient of this so-called kinetic contribution to the symmetry energy is $\epsilon_{F} / 3$, where $\epsilon_{F} \approx 28 \mathrm{MeV}$ is the Fermi energy. It amounts to only about $1 / 3$ of the symmetry term of $\approx 30 \mathrm{MeV}$ for nuclear matter at saturation. The major contribution is given by the potential term reflecting properties of the nuclear forces.

Microscopic many-body calculations have presented us with a variety of predictions for the nuclear equation of state $[10,12,64]$. The examples shown in fig. 4 for the two cases of symmetric nuclear matter and of pure neutron matter demonstrate that, overall, the results are quite compatible among each other, except for densities exceeding saturation for which the predictions diverge. The symmetry energy $E_{\text {sym }}$ is defined as the coefficient of the quadratic term in an expansion of the energy per particle 

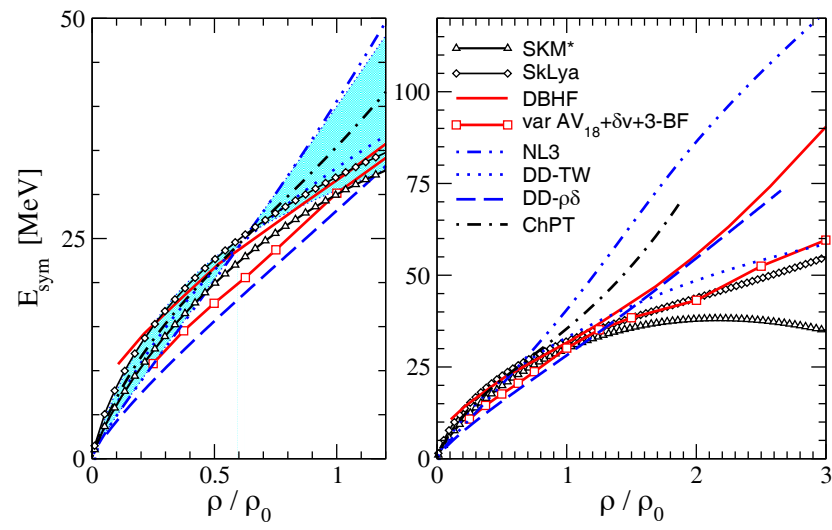

Fig. 5. Symmetry energy as a function of density as predicted by different models. The left panel zooms the low density range up to saturation. The full lines represent the DBHF and variational approaches using realistic forces (from ref. [10], reprinted with kind permission from Springer Science+Business Media).

in the asymmetry $\delta=\left(\rho_{n}-\rho_{p}\right) / \rho$, where $\rho_{n}, \rho_{p}$, and $\rho$ represent the neutron, proton, and total densities, respectively,

$$
E / A(\rho, \delta)=E / A(\rho, \delta=0)+E_{\mathrm{sym}}(\rho) \cdot \delta^{2}+\mathcal{O}\left(\delta^{4}\right) .
$$

In the usual quadratic approximation, the symmetry energy is the difference between the energies of neutron matter $(\delta=1)$ and symmetric matter $(\delta=0)$. As expected from fig. 4 , also the predictions for the symmetry energy diverge at high densities while most of them coincide near or slightly below saturation, the density range at which constraints from finite nuclei are valid (fig. 5).

In calculations using realistic forces fitted to two- and three-nucleon data, the uncertainty is mainly related to the short-range behavior of the nucleon-nucleon force and, in particular, to the three-body and tensor forces [20-22]. The three-body force has been shown to make an essential contribution of several $\mathrm{MeV}$ to the masses of light nuclei [65]. The extrapolation to higher densities of the partly phenomenological terms used there is, however, highly uncertain [21]. The general effect of including three-body forces in the calculations is a stiffening of the symmetry energy with increasing density [66-68]. Short-range correlations become also increasingly important at higher densities; results from very recent new experiments will, therefore, have a strong impact on predictions for high-density nuclear matter $[20,24,25]$.

It is also well known that nuclear mean fields are momentum dependent $[7,8,32,69]$. It is evident, e.g., in the energy dependence of the nuclear optical potential. The dominating effect is seen in the isoscalar sector but the isovector momentum dependence may also be important. It manifests itself as an energy dependence of the isospindependent part of the optical potential but can also be expressed in terms of a difference of the effective masses of protons and neutrons $[7,8,10]$. Even the ordering of these effective masses is still an open problem. It has, moreover, been shown that the effective mass differences and the asymmetry dependence of the EoS are both influencing particle yields and flow observables, and that additional observables will be needed to resolve the resulting ambiguity $[8,32,69]$.

Transport theory provides the tools for following the temporal evolution of nuclear reactions. For describing the composition-dependent part of the nuclear mean field, parameterizations based on potential models are commonly used. In the ultrarelativistic QMD (UrQMD) model of the group of $\mathrm{Li}$ and Bleicher [44,70,71], the potential part of the symmetry energy is defined with two parameters, the value at saturation density, usually taken as $22 \mathrm{MeV}$ in their calculations, and the power law coefficient $\gamma$ describing the dependence on density as $\left(\rho / \rho_{0}\right)^{\gamma}$. In the QMD model of the Tübingen group $[40,72,73]$ (both approaches are discussed in more detail in sect. 5), the nuclear potential of Das et al. with explicit momentum dependence in the isovector sector is used (MDI interaction, ref. [53]). There, as in the IBUU04 developed by the groups of $\mathrm{Li}$ and Chen $[7,54]$, the density dependence of the symmetry energy is characterized by a parameter $x$ appearing in the potential expressions. Examples of these parameterizations and of results obtained from the analysis of experimental reaction data are given in fig. 3 . The stiff $\left(E_{\mathrm{sym}}^{a}\right)$ and soft $\left(E_{\mathrm{sym}}^{b}\right)$ density dependences of fig. 2 correspond approximately to the cases $\gamma=1$ and $x=1$ shown there.

Parameterizations of this kind have the consequence that, once the symmetry energy at the saturation point is fixed, a single value at a different density or, alternatively, the slope or curvature at any density will completely determine the parameterization. Measurements of a variety of observables in nuclear structure and reactions have been used in this way to obtain results for the density dependence of the symmetry energy. They are often expressed in the form of the parameter $L$ which is proportional to the slope at saturation,

$$
L=3 \rho_{0} \cdot \mathrm{d} E_{\text {sym }} / \mathrm{d} \rho \mid \rho_{0} .
$$

Most results with their errors fall into the interval $20 \mathrm{MeV} \leq L \leq 100 \mathrm{MeV}$ and are compatible with a most probable value $L \approx 60 \mathrm{MeV}$, roughly corresponding to a power law coefficient $\gamma=0.6[7,16,17,52,74,75]$. The full line in fig. 3 represents, e.g., the result $\gamma=0.69$ deduced by $\mathrm{Li}$ and Chen from the MSU isospin-diffusion data and the neutron-skin thickness in ${ }^{208} \mathrm{~Pb}$ [54]. The corresponding slope parameter is $L=65 \mathrm{MeV}$. Rather similar constraints have been deduced from very recent investigations and observations of neutron-star properties [22,68,76-78]. Carefully constrained microscopic calculations with realistic potentials have also been shown to be fully compatible with these results as, e.g., $L=66.5 \mathrm{MeV}$ obtained by Vidaña et al. [79]. The present situation here as well as the information on the density dependence of the symmetry energy deduced from nuclear structure and reactions probing nuclear matter near and below saturation are given in the accompanying articles of this topical issue.

High expectations are placed on the determination of the neutron-skin thickness of ${ }^{208} \mathrm{~Pb}$ and other neutronrich nuclei by measuring the parity-violating contribution to electron scattering at high energy [80]. It will offer 
a practically model-free access to the slope at saturation, even though it is obtained by probing nuclear matter at an average density of typically $2 / 3$ of this value [81]. In the more distant future, gravitational wave detection may reach a sensitivity permitting the study of tidal deformations of neutron stars in coalescing binary systems, considered as strongly depending on the high-density symmetry energy [78].

\section{Directed and elliptic flows}

Collective flows have been known to be sensitive to essential features of the reaction dynamics for many years. Early studies have concentrated on the transition from mean-field dynamics to nucleon-nucleon collision dominated dynamics in the Fermi-energy domain [82, 83]. In experiments not sensitive to the absolute sign of the preferred direction of particle emission, it appeared as a socalled disappearance of flow, referring here to directed flow [84]. The corresponding energy, also called balance energy and observed at roughly 50 to $100 \mathrm{MeV}$ per nucleon, and its dependence on the mass and on the isotopic compositions of the colliding nuclei has attracted considerable interest [85-88]. Directed flow data, measured at even lower energies for selected isotopic and isobaric pairs of collision systems, have more recently been shown to provide information on the symmetry energy and its density dependence (refs. [89,90] and the accompanying article by Kohley and Yennello).

The influence of the density-dependent symmetry energy on the balance energy of heavy-collision systems from ${ }^{96} \mathrm{Zr}+{ }^{96} \mathrm{Zr}$ to ${ }^{197} \mathrm{Au}+{ }^{197} \mathrm{Au}$ has also been studied with the updated version of the UrQMD transport model [55]. It was found, e.g., that the balance energy of neutrons is particularly sensitive to the density dependence of the symmetry potential energy, an encouragement for new experiments since data of this kind do not exist up to now. In another systematic UrQMD study, the sign and magnitude of directed flow were shown to depend crucially on several of the many parameters entering the calculation [91]. To correctly reproduce the magnitude of directed $Z=1$ flow observed for semi-central ${ }^{197} \mathrm{Au}+{ }^{197} \mathrm{Au}$ collisions between 40 and $150 \mathrm{MeV}$ per nucleon, a careful adjustment of the isoscalar EoS and of the density and momentum dependence of the in-medium nucleon-nucleon elastic cross-sections had to be made. It emphasizes the need for consistent considerations of both, the mean field and the two-body collisions in transport models.

Reactions near the balance energy do not strongly compress the colliding matter. As shown above (fig. 1), several hundred $\mathrm{MeV}$ per nucleon up to $\approx 1 \mathrm{GeV}$ per nucleon are needed to reach densities of two to three times the saturation density in central collisions [5, 92]. At these energies, the pressure produced during the short high-density intervals initiates a collective outward motion whose strength will depend on the equation of state. The resulting direct and elliptic flows have been confronted with EoS model predictions and extreme assumptions have been shown to be ruled out [12].
Dynamical flow observables, expected to be influenced by the symmetry energy in asymmetric systems, have been proposed by several groups as probes for the equation of state at high density $[30,34-37]$. To be sensitive to the symmetry part, differential observables are required. The so-called differential neutron-proton flow is the difference of the parameters describing the collective motion of free neutrons and protons weighted by their numbers [30]. According to the simulations, this observable minimizes the influence of the isoscalar part in the EoS while maximizing that of the symmetry term [93]. Its proportionality to the particle multiplicities, however, makes its determination very dependent on the experimental efficiencies of particle detection and identification and on the precise procedure for distinguishing free and bound nucleons in calculations. Therefore, also differences or ratios of directed and elliptic flows have been considered.

It has become customary to express both, directed and elliptic flows, and possibly also higher flow components by means of a Fourier decomposition of the azimuthal distributions measured with respect to the orientation of the reaction plane $\phi_{R}[94-96]$,

$$
\frac{\mathrm{d} N}{\mathrm{~d}\left(\phi-\phi_{R}\right)}=\frac{N_{0}}{2 \pi}\left(1+2 \sum_{n \geq 1} v_{n} \cos n\left(\phi-\phi_{R}\right)\right),
$$

where $N_{0}$ is the azimuthally integrated yield. The coefficients $v_{n} \equiv\left\langle\cos n\left(\phi-\phi_{R}\right)\right\rangle$ are functions of particle type, impact parameter, rapidity $y$, and the transverse momentum $p_{t}$.

Excitation functions of the directed and elliptic flows for light charged particles from ${ }^{197} \mathrm{Au}+{ }^{197} \mathrm{Au}$ collisions are shown in figs. 6 and 7 . The directed flow is quantified as the derivative of $v_{1}$ with respect to rapidity, taken at mid-rapidity, and a positive slope, by definition, identifies a predominance of repulsion. The data for $Z=1$ and $Z=2$ particles illustrate the $Z$ dependence of the balance energy, being higher by more than $10 \mathrm{MeV}$ per nucleon for the hydrogen isotopes as compared to helium.

The excitation function of the elliptic flow, quantified as $v_{2}$ at midrapidity and shown in fig. 7 for $Z=1$ particles in ${ }^{197} \mathrm{Au}+{ }^{197} \mathrm{Au}$ collisions from various experiments, extends up to the AGS regime of several GeV per nucleon incident energies at which in-plane flow starts to dominate again. Squeeze-out perpendicular to the reaction plane $\left(v_{2}<0\right)$, as a result of shadowing by the spectator remnants is observed at incident energies between about $150 \mathrm{MeV}$ per nucleon and $4 \mathrm{GeV}$ per nucleon with a maximum near $400 \mathrm{MeV}$ per nucleon. At lower energies, the collective rotation in the mean-field dominated dynamics causes the observed in-plane enhancement of emitted reaction products [103]. The two figures also illustrate the precision that can be reached in flow measurements. The reliability of the applied methods is demonstrated by the good agreement of results from different experiments in the overlap regions of the studied intervals in collision energy $[98,99]$.

Elliptic flow has become an important observable at other energy regimes as well. At ultrarelativistic energies, 

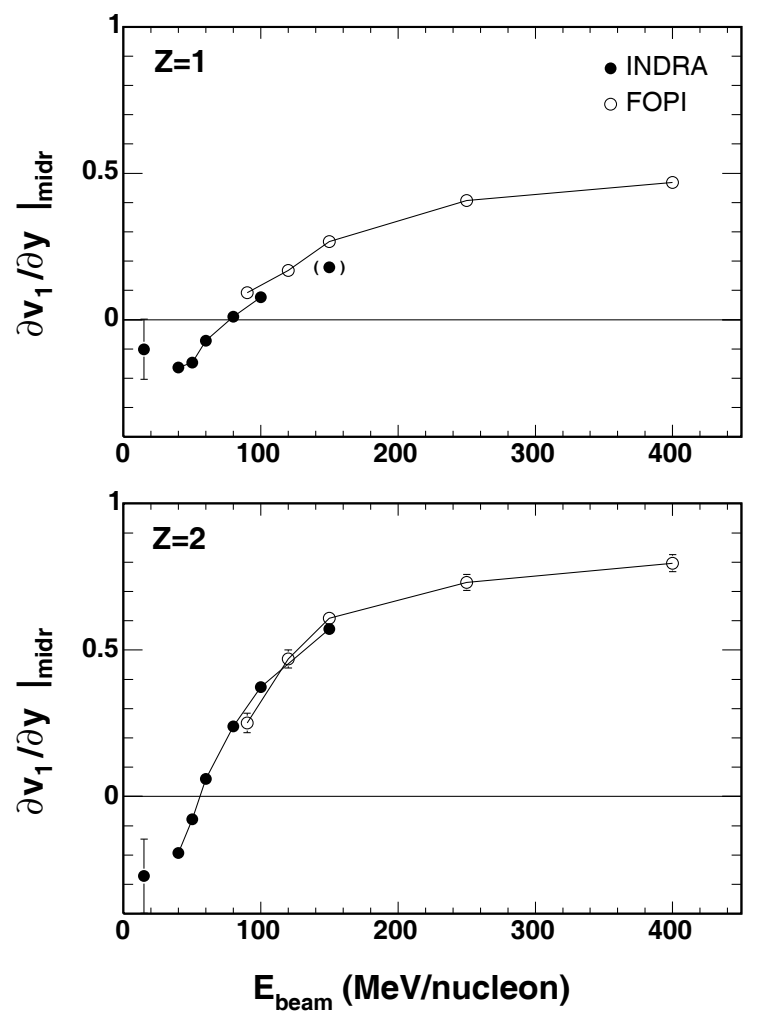

Fig. 6. Slopes of directed flow $\partial v_{1} / \partial y$ for $Z=1$ (top) and $Z=2$ (bottom) particles integrated over transverse momentum $p_{t}$ for mid-central ${ }^{197} \mathrm{Au}+{ }^{197} \mathrm{Au}$ collisions $(2-5.5 \mathrm{fm})$. The open and filled symbols represent the FOPI [97] and the INDRA data, respectively. The uncertainty at $15 \mathrm{MeV}$ per nucleon is mainly statistical. The INDRA point, in brackets, at $150 \mathrm{MeV}$ per nucleon in the top panel is biased due to experimental inefficiencies for $Z=1$ at this energy (from ref. [98], reprinted with kind permission from Springer Science+Business Media).

the observation of the constituent-quark scaling of elliptic flow is one of the prime arguments for deconfinement during the early collision phase, and properties of the formed quark-gluon liquid are deduced from the observed magnitude of collective motions $[1-4,104,105]$. It implies that elliptic flow develops very early in the collision which is valid also in the present range of relativistic energies as confirmed by calculations [11].

Experimentally, the azimuthal angle of emission is determined with respect to the orientation of a reaction plane that has been reconstructed from observed emission patterns. Several methods have been proposed (see, e.g., ref. [98]) which have in common an overall dependence of their accuracy on the emitted particle types and multiplicities of the considered reaction. The so-called dispersion of the reaction plane refers to the uncertainty of the reconstructed azimuthal orientation. The deduced flow parameters decrease in the case of poorly determined experimental reaction planes and corrections are necessary for which, however, quite refined methods exist. Their magnitude increases with the order of the Fourier coefficient considered.

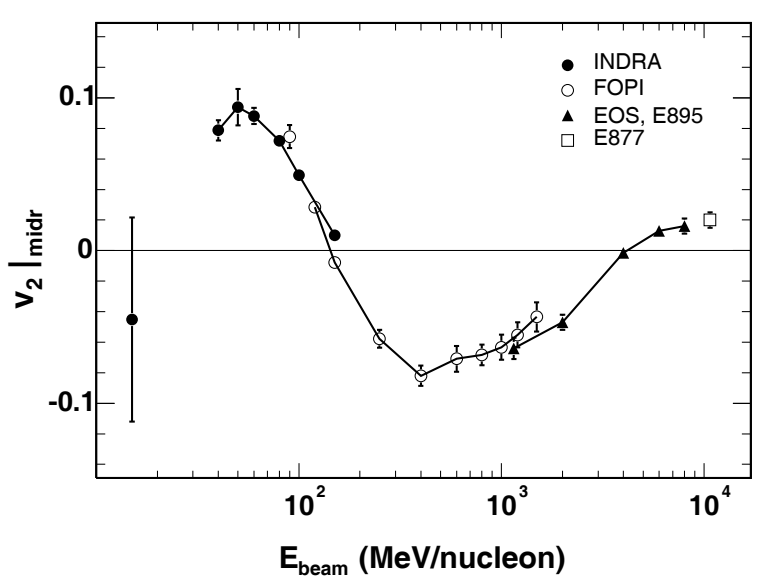

Fig. 7. Elliptic flow parameter $v_{2}$ at mid-rapidity for ${ }^{197} \mathrm{Au}+$ ${ }^{197} \mathrm{Au}$ collisions at intermediate impact parameters (about 5.5$7.5 \mathrm{fm}$ ) as a function of incident energy, in the beam frame. The filled and open circles represent the INDRA and FOPI data $[99,100]$, respectively, for $Z=1$ particles, the triangles represent the EOS and E895 data [101] for protons and the square represents the E877 data [102] for all charged particles (from ref. [98], reprinted with kind permission from Springer Science+Business Media).

In the INDRA and FOPI data sets included in the figures, the reaction plane has been reconstructed using the so-called Q-vector method in slightly different forms. The Q-vector representing the orientation of the reaction plane is calculated as the weighted sum of the transverse momenta of the measured reaction products with the weights chosen to be $+(-) 1$ for products in the forward (backward) c.m. hemisphere [106]. The obtained corrections are close to one, independent of the specific method, for the range of higher incident energies $(E>100 A \mathrm{MeV})$ where the directed flow is large and the reaction plane well defined by the high-multiplicity distribution of detected particles. At energies below $100 \mathrm{MeV}$ per nucleon, the inverse correction factors drop significantly and start to depend on the chosen method. The FOPI flow results, as published in refs. $[97,100]$ and shown in figs. 6 and 7 , have been corrected using the standard method. The midrapidity region of \pm 0.3 of the scaled c.m. rapidity has been excluded to improve the resolution. The corrections used for the INDRA data have been obtained with a new method adapted to the smaller multiplicities and increased emissions of intermediate mass fragments that are encountered at the lower energies $[98,107]$.

There exists a considerable amount of flow data in the literature with the potential of being useful for improving our understanding of the development of the observed collectivity during the high-density phase and its dependence on isospin. The FOPI Collaboration has published a comprehensive report on their measurements of azimuthal asymmetries in particle emissions in the regime of $1 \mathrm{GeV}$ per nucleon incident energies [29]. It includes data for the ${ }^{197} \mathrm{Au}+{ }^{197} \mathrm{Au}$ reaction from $90 \mathrm{MeV}$ to $1.5 \mathrm{GeV}$ per nucleon but also data for other systems. As an example, the elliptic flow of protons is shown in fig. 8 for the 


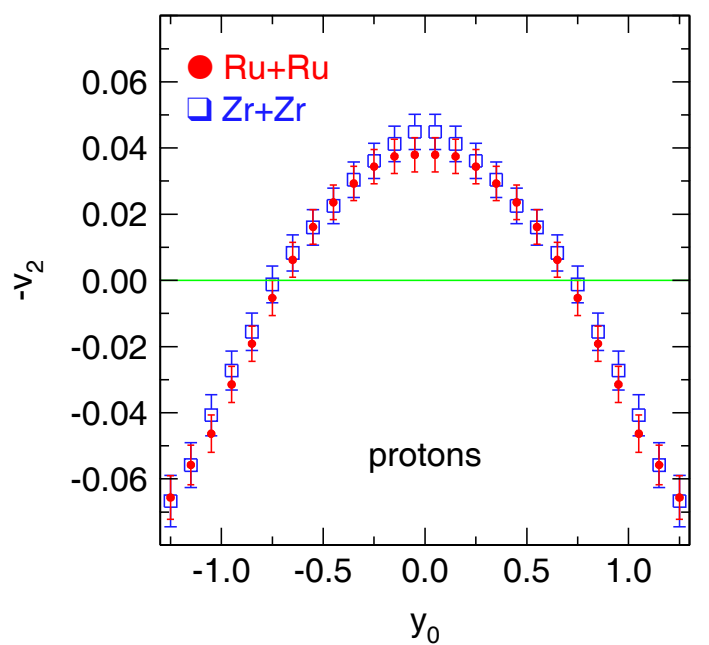

Fig. 8. Elliptic flow $-v_{2}\left(y_{0}\right)$ of protons in ${ }^{96} \mathrm{Zr}+{ }^{96} \mathrm{Zr}$ (blue open squares) and ${ }^{96} \mathrm{Ru}+{ }^{96} \mathrm{Ru}$ (red dots) collisions for $1.5 \mathrm{GeV}$ per nucleon incident energy and centrality $0.25<b_{0}<$ 0.45 . Note the inversion of $v_{2}$ and the definitions of the reduced impact parameter $b_{0}=b / b_{\max }$ and of the normalized rapidity in the c.m. frame, $y_{0}=y / y_{p}$, with $y_{p}$ denoting the projectile rapidity (reprinted from ref. [29], Copyright (2012), with permission from Elsevier).

isotopic pair of mass-symmetric systems ${ }^{96} \mathrm{Zr}+{ }^{96} \mathrm{Zr}$ and ${ }^{96} \mathrm{Ru}+{ }^{96} \mathrm{Ru}$ at the incident energy $1.5 \mathrm{GeV}$ per nucleon. It is interesting that, even with the precision achieved in this experiment, only small differences are visible, indicating a minute response to the differences of the corresponding mean fields. Similar observations have been made by the INDRA/ALADIN Collaboration studying several $\mathrm{Xe}+\mathrm{Sn}$ reactions at $100 \mathrm{MeV}$ per nucleon with ${ }^{124,129}$ Xe projectiles and ${ }^{112,124}$ Sn targets [108].

A complementary type of observable is represented by ratios of mirror nuclei and their flow properties. The example of ${ }^{3} \mathrm{H}$ and ${ }^{3} \mathrm{He}$ elliptic flows in ${ }^{197} \mathrm{Au}+{ }^{197} \mathrm{Au}$ collisions at 0.4 and $1.5 \mathrm{GeV}$ per nucleon incident energies demonstrates the rich information provided by exclusive measurements over wide ranges of rapidity and incident energies (fig. 9). The values of $v_{2}$ at midrapidity, their sign changes, and their behavior at the spectator rapidities are similar at the lower but significantly different at the higher energy. The discussion of their results by Reisdorf et al. includes the conjecture that, at the higher energy, momentum rather than density dependences may be responsible for the increased isotopic effect [29].

The extensive study of pion emission performed by the FOPI Collaboration contains, besides the pion yield ratios discussed in sect. 2, also data on pion flows [47]. The example of the impact parameter dependence of the elliptic flow of pions from ${ }^{96} \mathrm{Zr}+{ }^{96} \mathrm{Zr}$ and ${ }^{96} \mathrm{Ru}+{ }^{96} \mathrm{Ru}$ collisions at $1.5 \mathrm{GeV}$ per nucleon is shown in fig. 10. Squeeze-out dominates $\left(v_{2}<0\right)$ and its magnitude is significantly larger for the $\pi^{+}$than for the $\pi^{-}$case. It is also larger for the more proton-rich ${ }^{96} \mathrm{Ru}+{ }^{96} \mathrm{Ru}$ system, possibly indicating a significant role of Coulomb repulsion. A detailed discussion of the observed effects is presented, including comparisons with the Isospin-QMD (IQMD, ref. [109]) transport
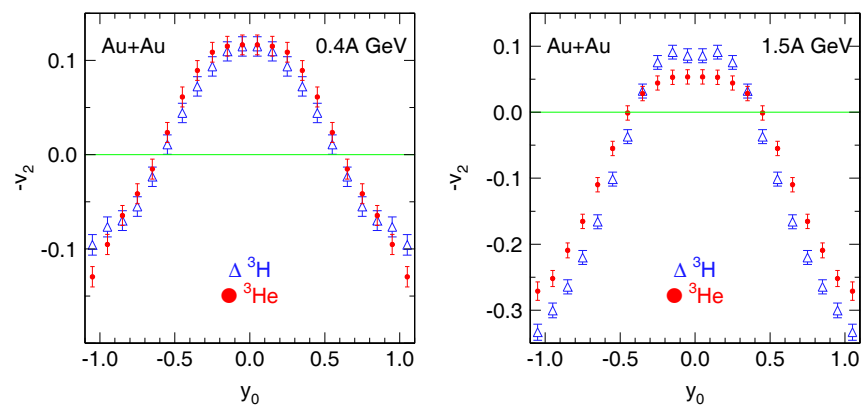

Fig. 9. Elliptic flow $-v_{2}\left(y_{0}\right)$ of ${ }^{3} \mathrm{H}$ (open triangles) and ${ }^{3} \mathrm{He}$ (filled circles) in ${ }^{197} \mathrm{Au}+{ }^{197} \mathrm{Au}$ collisions for 0.4 (left) and $1.5 \mathrm{GeV}$ per nucleon (right) incident energy and centrality $0.25<b_{0}<0.45$. Note the inversion of $v_{2}$ and the definitions of the reduced impact parameter $b_{0}=b / b_{\max }$ and of the normalized rapidity $y_{0}=y / y_{p}$ in the c.m. frame (reprinted from ref. [29], Copyright (2012), with permission from Elsevier).

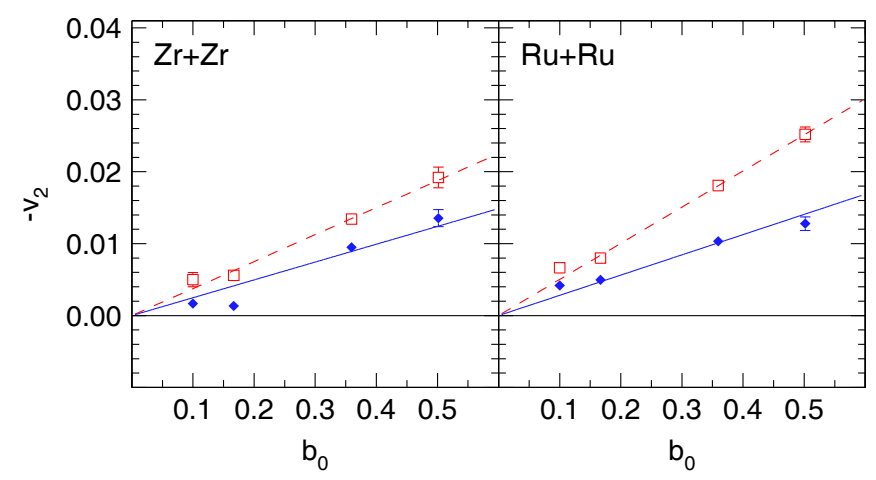

Fig. 10. Elliptic-flow parameter $-v_{2}$, integrated over rapidity $-1.8<y_{0}<0$, as a function of the reduced impact parameter $b_{0}=b / b_{\max }$ for the systems ${ }^{96} \mathrm{Zr}+{ }^{96} \mathrm{Zr}$ (left) and ${ }^{96} \mathrm{Ru}+{ }^{96} \mathrm{Ru}$ (right) at $1.5 \mathrm{GeV}$ per nucleon. Open squares and dashed lines represent the results for $\pi^{+}$while diamonds and full lines represent $\pi^{-}$. The lines are linear least square fits constrained to $v_{2}=0$ for $b_{0}=0$. Note the inversion of $v_{2}$ and the definition of the reduced rapidity $y_{0}=y / y_{p}$ in the c.m. frame (reprinted from ref. [47], Copyright (2007), with permission from Elsevier).

model which, however, did not fully describe the data. The authors, therefore, refrained from drawing definite conclusions on the EoS of nuclear matter and suggested the treatment of the $\Delta$ baryon propagation in the medium as an important topic for further studies. It is evident that collective flows may serve as an important complement to yield ratios in attempts to improve our understanding of the complex pion dynamics.

\section{Transport models and ingredients}

To study the sensitivity of the elliptic flow observables to the isospin-dependent part of the equation of state two independent transport models have been employed: UrQMD [44,70,71] and the Tübingen version of QMD [72, 73]. They have been upgraded to allow the study of the 
impact of the symmetry energy on observables that can be measured in intermediate-energy collisions of heavy ions.

\subsection{UrQMD}

This model, originally developed to study particle production at high energy [110], has been adapted to intermediate energy heavy-ion collisions by introducing a nuclear mean field corresponding to a soft EoS with momentum dependent forces, represented as two- and threebody Skyrme potentials supplemented by the long-range Yukawa and Coulomb interactions [111]. A new Pauliblocking scheme for the suppression of two-body collisions, which allows a better description of experimental observables at lower energies, has also been introduced [91]. Different options for the dependence on asymmetry were implemented, of which two are used here, expressed as a power law dependence of the potential part of the symmetry energy on the nuclear density $\rho$ according to

$$
\begin{aligned}
E_{\mathrm{sym}} & =E_{\mathrm{sym}}^{\mathrm{pot}}+E_{\mathrm{sym}}^{\mathrm{kin}} \\
& =22 \mathrm{MeV} \cdot\left(\rho / \rho_{0}\right)^{\gamma}+12 \mathrm{MeV} \cdot\left(\rho / \rho_{0}\right)^{2 / 3},
\end{aligned}
$$

with $\gamma=0.5$ and $\gamma=1.5$ corresponding to a soft and a stiff density dependence (fig. 3). The kinetic part remains unchanged.

The issue of the in-medium modification of nucleonnucleon cross-sections (NNCS) has been addressed by assuming that the elastic part can be factorized as a product of a medium correction factor $F(u, \alpha, p)$, with $u=\rho / \rho_{0}$, and the free elastic NNCS $\sigma_{e l}^{\text {free }}$,

$$
\sigma_{t o t}^{*}=\sigma_{i n}^{*}+\sigma_{e l}^{*}=\sigma_{i n}^{f r e e}+F(u, \alpha, p) \sigma_{e l}^{\text {free }} .
$$

The medium modification factor is proportional to both an isoscalar density effect $F_{u}$ and an isovector mass-splitting effect $F_{\alpha}$, which both should be functions of the relative momentum $p_{N N}$ of the two colliding particles in the $N N$ center-of-mass system. In refs. [71, 112], they have been parameterized as

$$
F_{\alpha, u}^{\mathrm{p}}= \begin{cases}f_{0} & p_{N N}>1 \mathrm{GeV} / c \\ \frac{F_{\alpha, u}-f_{0}}{1+\left(p_{N N} / p_{0}\right)^{\kappa}}+f_{0} & p_{N N} \leq 1 \mathrm{GeV} / c .\end{cases}
$$

The factor $F_{u}$ can be expressed as

$$
F_{u}=\lambda+(1-\lambda) \exp [-u / \zeta] .
$$

Here $\zeta$ and $\lambda$ are parameters which determine the density dependence of the cross-sections while $f_{0}, p_{0}$, and $\kappa$ in eq. (6) determine the restoration of the free cross-section with increasing relative momentum. Several parameter sets, shown in tables 1 and 2, have been selected to illustrate the resulting modifications of the NNCS (fig. 11).

The reduction of the elastic NNCS as a function of density becomes increasingly more pronounced as the parameterization is changed from FU1 to FU3. At the reduced density $u=2$, e.g., the values of FU1, FU2, and
Table 1. The three parameter sets FU1, FU2, and FU3 used for the density-dependent correction factor $F_{u}$ of elastic NNCS.

\begin{tabular}{|l|c|c|}
\hline Set & $\lambda$ & $\zeta$ \\
\hline \hline FU1 & $1 / 3$ & 0.54568 \\
FU2 & $1 / 4$ & 0.54568 \\
FU3 & $1 / 6$ & $1 / 3$ \\
\hline
\end{tabular}

Table 2. The three parameter sets FP1, FP2, and FP3 used for describing the momentum dependence of $F_{u}$. The fourth case, without a $p_{N N}$ limit, is obtained by setting $f_{0}$ equal to $F(u)$ in eq. (6).

\begin{tabular}{|l|c|c|c|}
\hline Set & $f_{0}$ & $p_{0}\left[\mathrm{GeV} \mathrm{c} c^{-1}\right]$ & $\kappa$ \\
\hline \hline FP1 & 1 & 0.425 & 5 \\
FP2 & 1 & 0.225 & 3 \\
FP3 & 1 & 0.625 & 8 \\
\hline no $p_{N N}$ limit & $F(u)$ & $/$ & $/$ \\
\hline
\end{tabular}

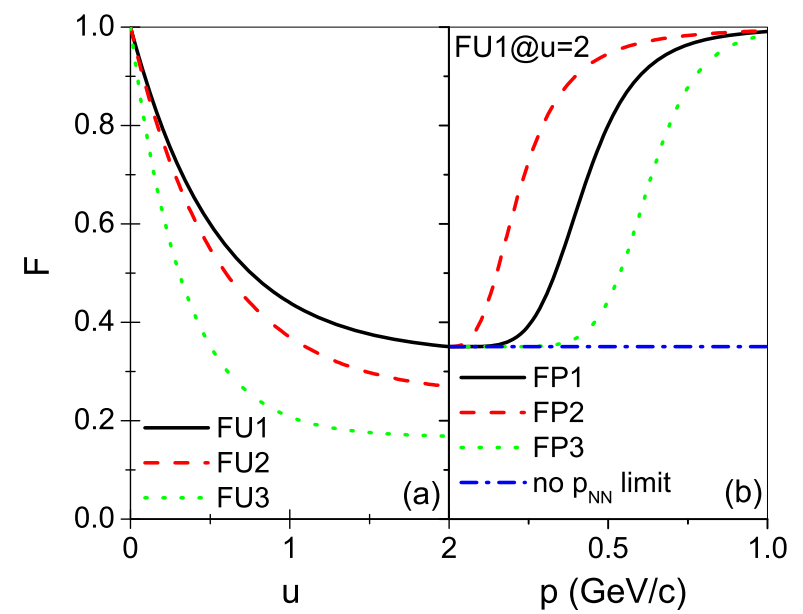

Fig. 11. (a) Correction factor $F$ obtained with the parameterizations FU1, FU2, and FU3 given in table 1 and (b) the momentum dependence obtained with the four options FP1, FP2, FP3, and no pNN limit given in table 2 for the example of FU1 at $u=2$ (reprinted with permission from ref. [91]; Copyright (2011) by the American Physical Society).

FU3 are $0.35,0.27$, and 0.17 , respectively. We note that the density dependence of the FU1 parameterization is in qualitative agreement with previous work based on the Dirac-Brueckner approach [113-115]. However, in a previous investigation of the elastic NNCS, based on the effective Lagrangian of density-dependent relativistic hadron theory in which the $\sigma, \omega, \rho$ and $\delta\left[a_{0}(980)\right]$ mesons are included [116], it was shown that especially the neutronproton cross-sections $\sigma_{e l, n p}^{*}$ might be largely reduced in the neutron-rich nuclear medium; the corresponding reduction factor might be as low as $\sim 0.1$ at $u=2$. Therefore, the other parameter sets FU2 and FU3 (table 1) are still to be considered reasonable assumptions. The importance of the elastic NNCS for describing collective flows in this energy range has been confirmed by a study using the 
recently developed version of the UrQMD model in which the Skyrme potential energy density functional has been introduced [117].

The UrQMD transport program is stopped at a collision time of $150 \mathrm{fm} / c$ at which point a conventional phase-space coalescence model with two parameters is used to construct clusters. Nucleons with relative momenta smaller than $P_{0}$ and relative distances smaller than $R_{0}$ are considered as belonging to the same cluster. The values $P_{0}=0.275 \mathrm{GeV} / c$ and $R_{0}=3.0 \mathrm{fm}$ have been adopted as standard parameters. With these values the overall dependence of cluster yields on $Z$ is rather well reproduced but the yields of $Z=2$ particles are underpredicted by a factor of 3 . The yields of deuterons and tritons in central collisions are also underestimated by similar factors [39].

According to the UrQMD calculations, one of the most promising probes of the strength of the symmetry energy at supra-saturation densities is the difference of the neutron and proton (or hydrogen) elliptic flows. The predictions for the parameter $v_{2}$ of emitted neutrons, protons, and hydrogen isotopes for mid-peripheral ${ }^{197} \mathrm{Au}+{ }^{197} \mathrm{Au}$ collisions at $400 \mathrm{MeV}$ per nucleon and for the two choices of the density dependence of the symmetry energy, labeled asy-stiff $(\gamma=1.5)$ and asy-soft $(\gamma=0.5)$, are shown in fig. 12. They are displayed as a function of the laboratory rapidity $y_{\text {lab }}$. The dominant difference is the significantly larger neutron squeeze-out in the asy-stiff case as compared to the asy-soft case. The proton and hydrogen flows respond only weakly, and in opposite direction, to the variation of $\gamma$ within the chosen stiffness interval. Relative to each other, the neutron and proton elliptic flows vary on the level of $15 \%$. Their absolute magnitude is satisfactorily reproduced with the FP1 parameterization, as shown by the comparison with the FOPI experimental result for $Z=1$ particles at mid-rapidity (fig. 12). Note, however, that this depends sensitively on the chosen parameters as discussed in refs. [39, 91, 117].

The UrQMD model in the version described here has been employed, with minor changes, to study the impact of the isovector part of the equation of state of nuclear matter on a few other observables that can in principle be measured in heavy-ion collisions or other closely related topics: the $\Sigma^{-} / \Sigma^{+}$and $\pi^{-} / \pi^{+}$multiplicity ratios [118], the transverse momentum distribution of the elliptic flow difference [70], the beam-energy and impact-parameter dependences of the slope parameter of the double neutronto-proton ratio as a function of rapidity [31], the balance energies of free neutrons $[55,119]$ and the momentum dependence of the medium-modified nucleon-nucleon crosssections $[91,112]$.

\subsection{Tübingen QMD}

To study the impact of the symmetry energy term on elliptic flow observables, the Tübingen QMD model $[72,73]$ has been expanded by adding an isospin-dependent part to the mean-field potential $[40,58]$. Two possible parameterizations have been implemented: a momentum-dependent

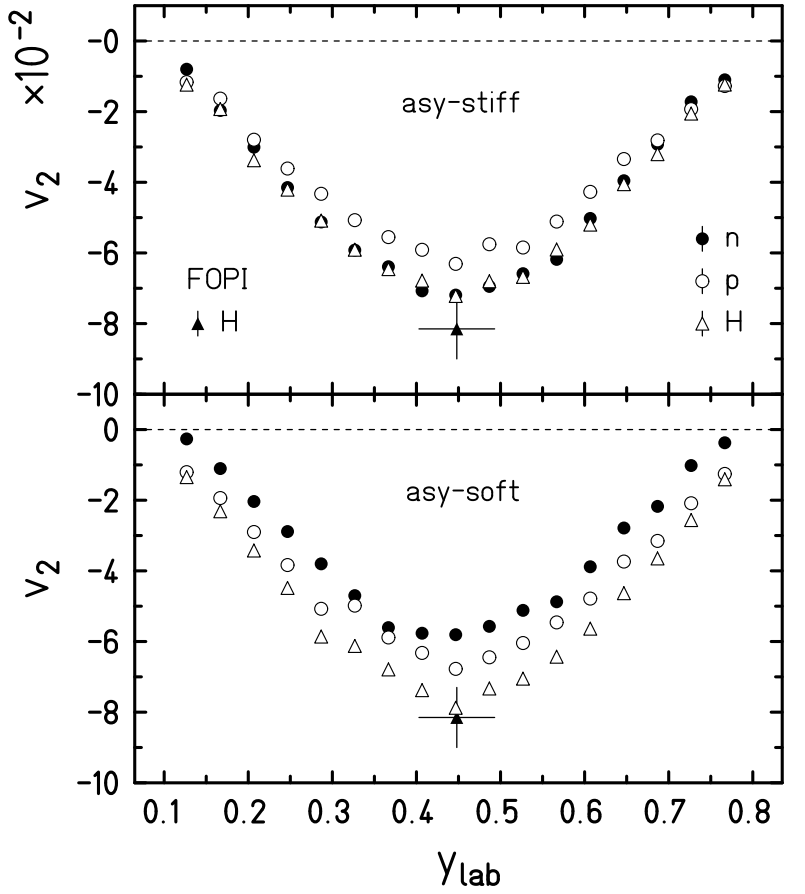

Fig. 12. Elliptic flow parameter $v_{2}$ for mid-peripheral $(5.5 \leq$ $b \leq 7.5 \mathrm{fm}){ }^{197} \mathrm{Au}+{ }^{197} \mathrm{Au}$ collisions at $400 \mathrm{MeV}$ per nucleon as calculated with the UrQMD model for neutrons (dots), protons (circles), and all hydrogen isotopes ( $Z=1$, open triangles), integrated over transverse momentum $p_{t}$, as a function of the laboratory rapidity $y_{1 \mathrm{ab}}$. The predictions obtained with a stiff and a soft density dependence of the symmetry term are given in the upper and lower panels, respectively. The experimental result from ref. [100] for $Z=1$ particles at mid-rapidity is represented by the filled triangle (reprinted from ref. [39], Copyright (2011), with permission from Elsevier).

version which has been developed in ref. [53] starting from the Gogny effective interaction,

$$
\begin{aligned}
U(\rho, \beta, p, \tau, x)= & A_{u}(x) \frac{\rho_{\tau^{\prime}}}{\rho_{0}}+A_{l}(x) \frac{\rho_{\tau}}{\rho_{0}} \\
& +B\left(\rho / \rho_{0}\right)^{\sigma}\left(1-x \beta^{2}\right) \\
& -8 \tau x \frac{B}{\sigma+1} \frac{\rho^{\sigma-1}}{\rho_{0}^{\sigma}} \beta \rho_{\tau^{\prime}} \\
& +\frac{2 C_{\tau \tau}}{\rho_{0}} \int \mathrm{d}^{3} p^{\prime} \frac{f_{\tau}\left(\boldsymbol{r}, \boldsymbol{p}^{\prime}\right)}{1+\left(\boldsymbol{p}-\boldsymbol{p}^{\prime}\right)^{2} / \Lambda^{2}} \\
& +\frac{2 C_{\tau \tau^{\prime}}}{\rho_{0}} \int \mathrm{d}^{3} p^{\prime} \frac{f_{\tau^{\prime}}\left(\boldsymbol{r}, \boldsymbol{p}^{\prime}\right)}{1+\left(\boldsymbol{p}-\boldsymbol{p}^{\prime}\right)^{2} / \Lambda^{2}},
\end{aligned}
$$

and a power law parameterization, momentum independent and similar to that used in ref. [39], which has been extended to allow also soft and super-soft scenarios [58],

$$
S(\rho)= \begin{cases}S_{0}\left(\rho / \rho_{0}\right)^{\gamma} & \text {-linear or stiff } \\ a+(18.5-a)\left(\rho / \rho_{0}\right)^{\gamma} & \text {-soft or super-soft. }\end{cases}
$$

The sets $a=23.0 \mathrm{MeV}, \gamma=1.0$ and $a=31.0 \mathrm{MeV}$, $\gamma=2.0$ in the lower parameterization reproduce a soft 
and super-soft density dependence, respectively. This allowed to test the dependence of the constraints extracted for the symmetry energy on its various parameterizations employed in the literature.

To study the model dependence of elliptic-flow observables, an explicit dependence of the microscopic nucleonnucleon cross-sections on density and isospin asymmetry has been introduced. The parameterization of the density dependence of elastic proton-proton and neutron-proton cross-sections below the pion production threshold obtained by Li and Machleidt $[113,114]$ is used. The isospin asymmetry dependence is introduced indirectly through a dependence of the in-medium nucleon masses on this parameter [54]. This approach is used also above the pion production threshold to simulate the dependence of nucleon-nucleon cross-sections on both density and isospin asymmetry.

The nucleon optical potential is an important ingredient of transport models which still bears some uncertainty on its magnitude. Its strength can be inferred from different sources: either from first principles [120] or from the experimental data of proton scattering on $\mathrm{Ca}$ and heavier nuclei within a relativistic description based on the Dirac-equation which in turn allowed the extraction of the momentum dependence of the bare interaction [121]. The results of the two approaches are somewhat different. The Brueckner-Hartree-Fock approach and its relativistic counterpart favor a potential that is attractive at all values of the momentum, while the relativistic Dirac approach delivers a potential that becomes repulsive above a certain momentum threshold, depending on which experimental data sets are considered. The Brueckner-HartreeFock approach, in addition, predicts an optical potential that is almost momentum independent at moderate values of the momentum.

To account for this model dependence, heavy-ion collisions have been simulated by considering three different parameterizations of the optical potential. The first one stems from the isoscalar part of the Gogny interaction [53] while the last two mimic the parameterizations presented in ref. [121]

$$
\begin{aligned}
V_{o p t}^{(M D I)} & =\left(C_{l}+C_{u}\right) \frac{1}{1+\left(\boldsymbol{p}_{\boldsymbol{i}}-\boldsymbol{p}_{\boldsymbol{j}}\right)^{2} / \Lambda^{2}} \frac{\rho_{i j}}{\rho_{0}} \\
V_{o p t}^{(H A)} & =\left\{V_{0}+v \ln ^{2}\left[a\left(\boldsymbol{p}_{\boldsymbol{i}}-\boldsymbol{p}_{\boldsymbol{j}}\right)^{2}+1\right]\right\} \frac{\rho_{i j}}{\rho_{0}} .
\end{aligned}
$$

The values of the parameters can be found in ref. [58] for all the cases presented in this review.

The QMD program is stopped at $t=60 \mathrm{fm} / c$, i.e. also long after the high-density phase of the reaction (fig. 1). In order to extract the spectra of free nucleons, a density cutoff at $\rho_{0} / 8$ is applied. The degree of clusterization, as evident from the FOPI experimental data, is underestimated by this procedure, however. A fine tuning of the density cutoff parameter can improve in this respect but the theoretical estimates for the elliptic flows of free nucleons depend only mildly on it.

In addition to the already mentioned transport model ingredients, the study performed by Cozma [40] has also

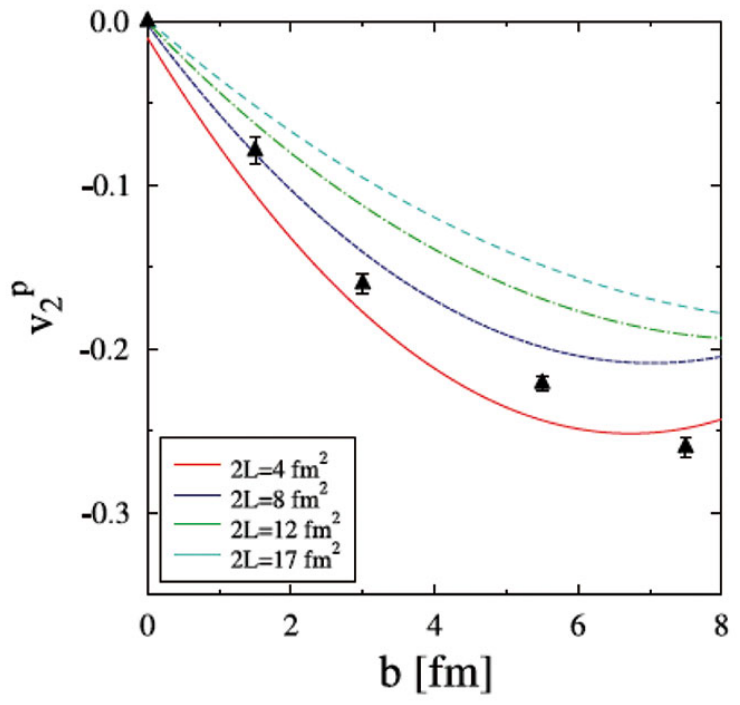

Fig. 13. Elliptic flow of protons as a function of the impact parameter for various assumptions for the width of the nucleon wave packet. Note that there are factors of 2 difference between $v_{2}$ and $L$ as defined in ref. [40] and here; the ordinate $v_{2}^{p}$ of the figure is equal to $2 \cdot v_{2}^{p}$ as defined in eq. (3) and the values of $2 L$ in the legend correspond to the same values of $L$ discussed in the text (reprinted from ref. [40], Copyright (2011), with permission from Elsevier).

addressed the impact of other model parameters on elliptic flow observables, in particular those of the nuclear matter compressibility modulus $K$ and of the width $L$ of the nucleon wave function. The values of these two parameters have been varied around those commonly accepted in the literature, $K=210 \mathrm{MeV}$ and $L=8.66 \mathrm{fm}^{2}$ [122]. It was found that the individual elliptic flows of neutrons or protons show a rather sizable sensitivity to the parameter that is varied. This is presented in fig. 13 for the case of $L$ which is varied between the extremes $L=4.33 \mathrm{fm}^{2}$ and $L=17 \mathrm{fm}^{2}$. The former is used when simulating collisions of light nuclei while the latter represents a nucleon radius that is unrealistically large. The value $L=8.66 \mathrm{fm}^{2}$ is the standard choice used in simulations of collisions of heavy ions, like ${ }^{197} \mathrm{Au}$, as it improves the stability of static properties of the respective nuclei.

In contrast, the impact of model parameter changes on the difference of neutron and proton elliptic flows was found to be considerably smaller. This is exemplified in fig. 14 by plotting the variation of the neutron-proton elliptic flow difference to changes of the compressibility modulus from soft to hard, in comparison to variations due to modifications of the stiffness of the symmetry energy from super-stiff to super-soft. Similar statements hold true also for the case of the width $L$ of the nucleon wave function and for various scenarios for the in-medium nucleonnucleon cross-sections [40].

The Tübingen QMD transport model has been successfully applied to the description of several heavy-ion related topics in the $\mathrm{GeV}$ per nucleon regime of collision energies: dilepton emission [123-125], stiffness of the equation of state of symmetric nuclear matter [14] and various 


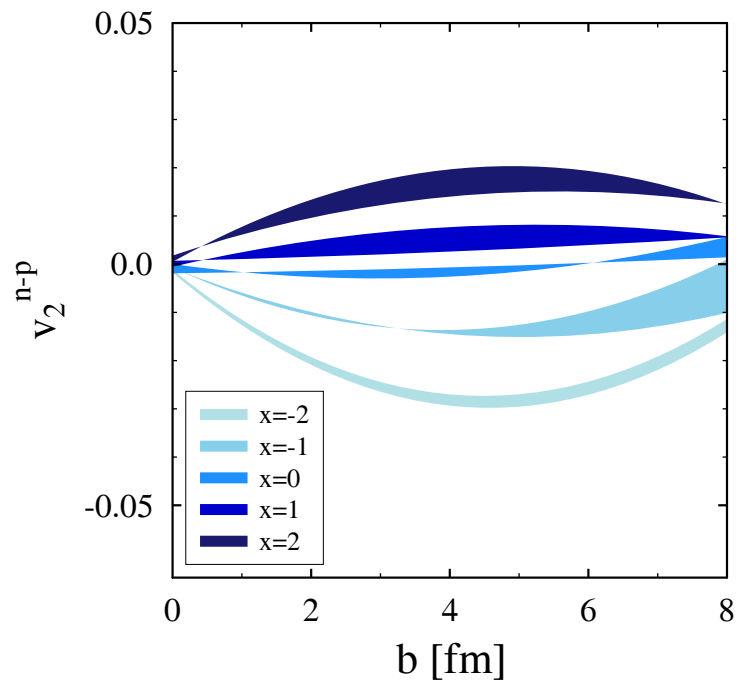

Fig. 14. Sensitivity of the neutron-proton elliptic flow difference $v_{2}^{n-p}=v_{2}^{n}-v_{2}^{p}\left(v_{2}\right.$ as defined in eq. (3)) to the isospinindependent EoS and to the choice of the symmetry term in the parameterization of ref. [54] as indicated. The widths of the bands represent the uncertainty arising from using either a soft ( $K=210 \mathrm{MeV}$ with momentum dependence) or a hard $(K=380 \mathrm{MeV})$ version for the isospin-independent part of the EoS. The FOPI acceptance filter has been applied to simulated data (reprinted from ref. [40], Copyright (2011), with permission from Elsevier).

in-medium effects relevant for the dynamics of heavy-ion collisions $[73,126]$.

\section{The FOPI/LAND experiment}

The squeeze-out of neutrons has first been observed by the FOPI/LAND Collaboration who studied the reaction ${ }^{197} \mathrm{Au}+{ }^{197} \mathrm{Au}$ at $400 \mathrm{MeV}$ per nucleon [41]. The squeezeout of charged particles reaches its maximum at this energy (fig. 7), and similarly large anisotropies were observed for neutrons [42]. The neutrons have been detected with the Large-Area Neutron Detector, LAND [43], while the FOPI Forward Wall, covering the forward range of laboratory angles $\theta_{\text {lab }} \leq 30^{\circ}$ with more than 700 plastic scintillator elements, was used to determine the modulus and azimuthal orientation of the impact parameter. LAND had been divided into two separate units which were placed next to each other at sideways positions, providing the kinematic acceptance shown in fig. 15.

The UrQMD results presented in the previous section provided the motivation for returning to the existing data set (fig. 12). A re-analysis has been performed which consisted mainly in choosing equal acceptances for neutrons and hydrogen isotopes with regard to particle energy, rapidity and transverse momentum (energy and momentum per nucleon for deuterons and tritons). The results obtained for a mid-peripheral event class are shown in fig. 16 as a function of the rapidity $y$, normalized with respect to the projectile rapidity $y_{p}=0.896$. Their asymmetry with

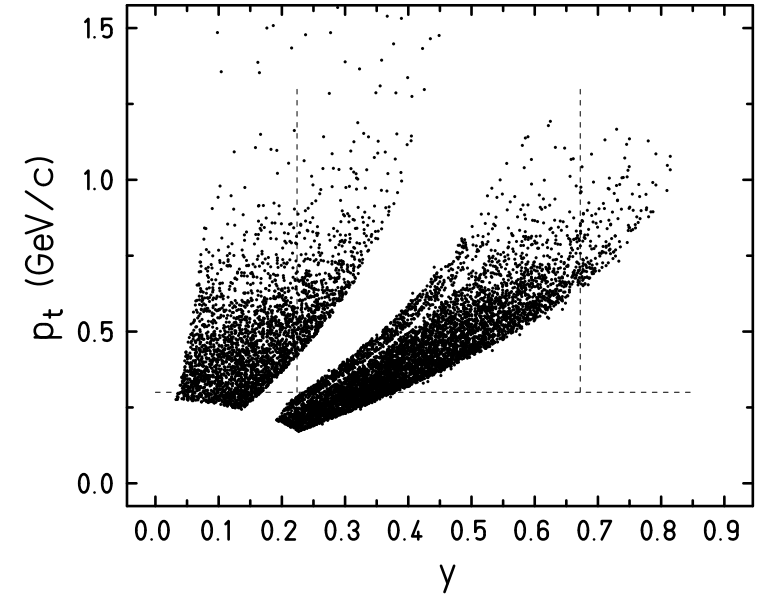

Fig. 15. Scatter plot of 10000 neutron events with energy $E_{\text {lab }} \geq 40 \mathrm{MeV}$ in the plane of transverse momentum $p_{t} v s$. rapidity $y$ in the laboratory frame from a run without shadow bars. The quality criteria are the same as used in the flow analysis. Their application produces the local inefficiency in the forward detector unit caused by individual detector modules with reduced performance. The dashed lines represent the acceptance cuts $p_{t} \geq 0.3 \mathrm{GeV} / c$ and $0.25 \leq y / y_{p} \leq 0.75$ applied in the analysis (reprinted from ref. [39], Copyright (2011), with permission from Elsevier).

respect to mid-rapidity, $y / y_{p}=0.5$, is caused by the kinematic acceptance of LAND (fig. 15). Its increase in $p_{t}$ with $y$ and the decreasing yields at large $p_{t}$ are responsible for the significant statistical errors at forward rapidity. The theoretical predictions have been obtained simulating the LAND acceptance and the experimental analysis conditions. The results, shown for neutrons in fig. 16, follow qualitatively the experimental data for both, the directed and elliptic flows. However, in contrast to the elliptic flow, the sensitivity of the directed flow of neutrons to the stiffness of the symmetry energy is predicted to be nearly negligible by the UrQMD (fig. 16, top panel).

The dependence of the elliptic flow parameter $v_{2}$ on the transverse momentum per nucleon, $p_{t} / A$, is shown in fig. 17, upper panel, for the full statistics of central and mid-peripheral collisions $(b \leq 7.5 \mathrm{fm})$ collected in this experiment. The measured values are approximately reproduced by the UrQMD predictions which are significantly different for the stiff $(\gamma=1.5)$ and soft $(\gamma=0.5)$ density dependences. For the quantitative comparison, the ratio of the flow parameters of neutrons vs. protons or vs. $Z=1$ particles has been proposed as a useful observable [39]. This choice is expected to minimize systematic effects influencing the collective flows of neutrons and charged particles in similar ways. This includes also technical issues as, e.g., the matching of the impact-parameter intervals used in the calculations with the corresponding experimental event groups or the magnitude of the corrections needed to account for the dispersion of the reaction plane. To test whether this kind of insensitivity applies also to model parameters in the isoscalar sector, the calculations were performed with the two parameterizations FP1 and FP2 of 


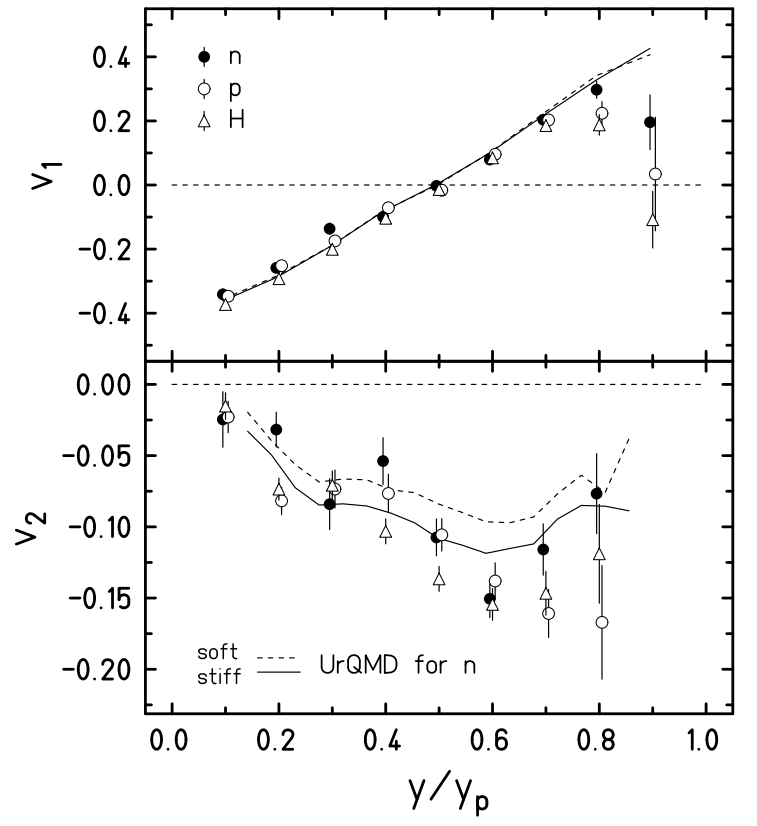

Fig. 16. Measured flow parameters $v_{1}$ (top) and $v_{2}$ (bottom) for mid-peripheral $(5.5 \leq b \leq 7.5 \mathrm{fm}){ }^{197} \mathrm{Au}+{ }^{197} \mathrm{Au}$ collisions at $400 \mathrm{MeV}$ per nucleon for neutrons (dots), protons (circles), and hydrogen isotopes $(Z=1$, open triangles) integrated within $0.3 \leq p_{t} / A \leq 1.3 \mathrm{GeV} / c$ per nucleon as a function of the normalized rapidity $y / y_{p}$. The UrQMD predictions for neutrons are shown for the FP1 parameterization of the in-medium cross-sections and for a stiff $(\gamma=1.5$, full lines) and a soft $(\gamma=0.5$, dashed) density dependence of the symmetry term. The experimental data have been corrected for the dispersion of the reaction plane (reprinted from ref. [39], Copyright (2011), with permission from Elsevier).

the momentum dependence of the elastic nucleon-nucleon cross-sections (cf. fig. 11). Their absolute predictions of $v_{2}$ at mid-rapidity differ by $\approx 40 \%$ for this reaction $[91,112]$.

In fig. 17 (lower panels), the results for the ratio with respect to the total hydrogen yield are shown. The calculated ratios exhibit clearly the sensitivity of the elliptic flow to the stiffness of the symmetry energy predicted by the UrQMD but depend only weakly on the chosen parameterization for the in-medium nucleon-nucleon crosssection. The experimental ratios, even though associated with large errors, scatter within the interval given by the two calculations. Linear interpolations between the predictions, averaged over $0.3<p_{t} / A \leq 1.0 \mathrm{GeV} / c$, yield very similar results $\gamma=1.01 \pm 0.21$ and $\gamma=0.98 \pm 0.35$ (standard deviations) for the two parameterizations. The error is larger for FP2 because the sensitivity is somewhat smaller.

This analysis was repeated in various forms. With the squeeze-out ratios $v_{2}^{n} / v_{2}^{p}$ of neutrons with respect to free protons, similar results were obtained, however with larger errors. The study of the impact parameter dependence indicated a slightly smaller value $\gamma \approx 0.5$ for the midperipheral event group, again with larger errors. It was also tested to which density region around $\rho_{0}$ the ellipticflow ratios are sensitive, with the result that both, sub-

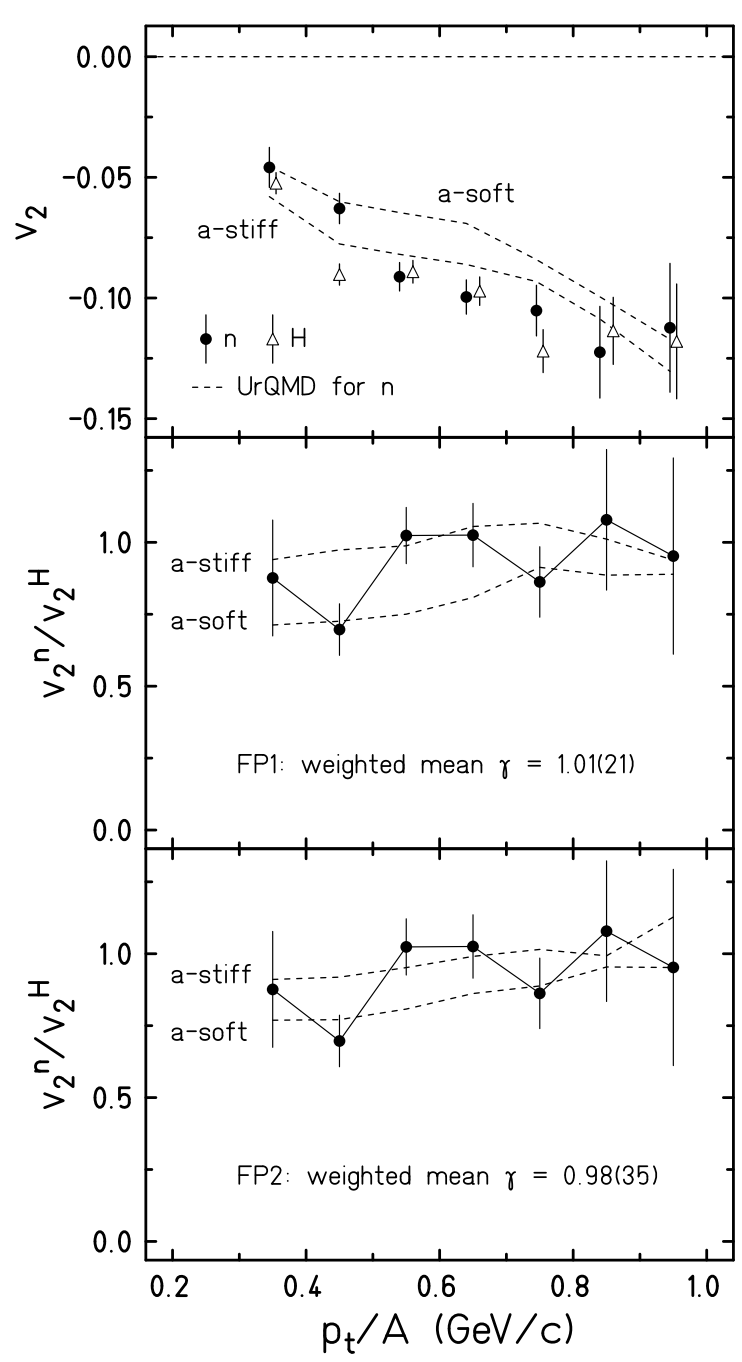

Fig. 17. Elliptic flow parameters $v_{2}$ for neutrons (dots) and hydrogen isotopes (open triangles, top panel) and their ratio (lower panels) for moderately central $(b<7.5 \mathrm{fm})$ collisions of ${ }^{197} \mathrm{Au}+{ }^{197} \mathrm{Au}$ at $400 \mathrm{MeV}$ per nucleon, integrated within the rapidity interval $0.25 \leq y / y_{p} \leq 0.75$, as a function of the transverse momentum per nucleon $p_{t} / A$. The symbols represent the experimental data. The UrQMD predictions for $\gamma=1.5$ (a-stiff) and $\gamma=0.5$ (a-soft) obtained with the FP1 parameterization for neutrons (top panel) and for the ratio (middle panel), and with the FP2 parameterization for the ratio (bottom panel) are given by the dashed lines (reprinted from ref. [39], Copyright (2011), with permission from Elsevier).

and supra-saturation densities are probed with this observable [39].

In consideration of the apparent systematic and experimental errors, a value $\gamma=0.9 \pm 0.4$ has been adopted by the authors as best representing the power-law exponent of the potential term resulting from the elliptic-flow analysis. It falls slightly below the $\gamma=1.0$ line shown in fig. 3 but, with the quoted uncertainty, stretches over the interval from $\gamma=0.5$ halfway up to $\gamma=1.5$. The corresponding slope parameter is $L=83 \pm 26 \mathrm{MeV}$. According to the UrQMD, the squeeze-out data indicate a moderately 


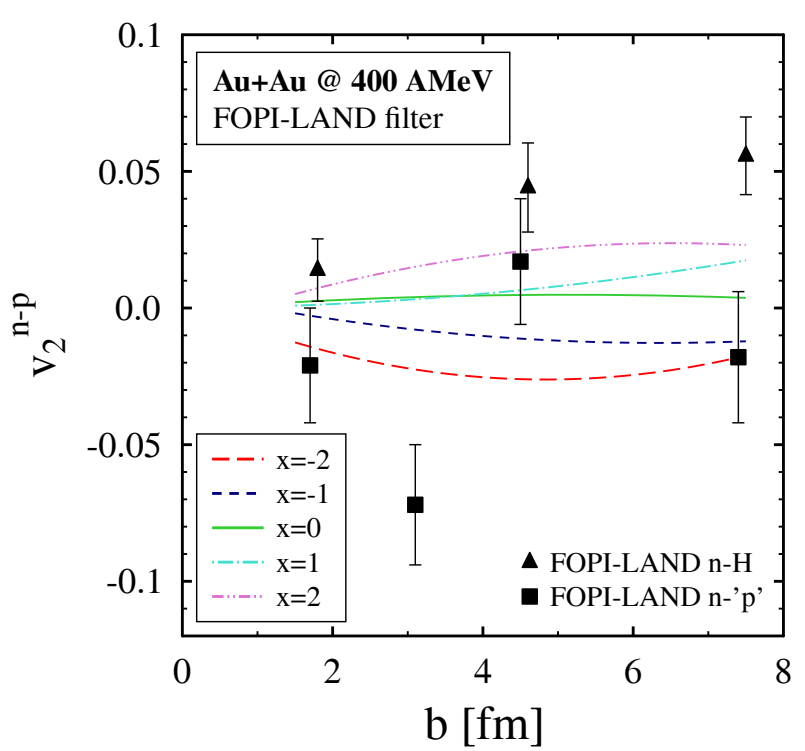

Fig. 18. Comparison between theoretical estimates for the neutron-proton elliptic flow difference and the FOPI-LAND experimental data for the neutron-proton and neutron-hydrogen elliptic flow differences. Note that the $v_{2}$ parameter displayed here is twice larger than the $v_{2}$ according to eq. (3) because of a factor of 2 difference in the definitions used in ref. [40] and here (reprinted from ref. [40], Copyright (2011), with permission from Elsevier).

soft to linear behavior of the symmetry energy that is consistent with the density dependence deduced from experiments probing nuclear matter near or below saturation. Comparing with the many-body theories shown in fig. 5 , the elliptic-flow result is in good qualitative agreement with the range spanned by the DBHF and variational calculations based on realistic nuclear potentials.

In an independent analysis, Cozma has used data from the same experiment and investigated the influence of several parameters on the difference between the elliptic flows of protons and neutrons using the Tübingen version of the QMD transport model (ref. [40] and sect. 5.2). They included the parameterization of the isoscalar EoS, the choice of various forms of free or in-medium nucleonnucleon cross-sections, and model parameters as, e.g., the widths of the wave packets representing nucleons. The interaction developed by Das et al. was used which contains an explicit momentum dependence of the symmetry energy part $[53,54]$. Experimental data for $v_{2}^{p}$ presented in ref. [42] show a scattered dependence on the impact parameter and could not be used to convincingly constrain the stiffness of the symmetry energy. However, the hydrogen elliptic flow parameter $v_{2}^{H}$ published in ref. [41] does not suffer from such a problem and, in view of the fact that it represents an upper value (in absolute magnitude) for $v_{2}^{p}$, it can be used to constrain the high-density dependence of the symmetry energy via the neutron-hydrogen elliptic flow difference. As concluded by Cozma [40], an upper limit on the softness of the symmetry energy is obtained from the comparison with the experimental flow data, as can be inferred from fig. 18 .

\section{The ASY-EOS experiment}

The ASY-EOS experiment, conducted at the GSI laboratory in 2011, represents an attempt to considerably improve the statistical accuracy of the flow parameters for the ${ }^{197} \mathrm{Au}+{ }^{197} \mathrm{Au}$ system at $400 \mathrm{MeV}$ per nucleon but also to complement these measurements with other observables and data for other systems [59,60]. Additional constraints for the comparison with transport models were considered to be useful for narrowing down the uncertainties of their predictions as shown in the following section. For this purpose, data were collected for two additional systems, the neutron-rich ${ }^{96} \mathrm{Zr}+{ }^{96} \mathrm{Zr}$ and neutron-poor ${ }^{96} \mathrm{Ru}+{ }^{96} \mathrm{Ru}$ pair of mass-symmetric $A=96$ collision systems, both also at $400 \mathrm{MeV}$ per nucleon incident energy. The FOPI Collaboration has studied these three reactions in quite some detail, at the present energy $400 \mathrm{MeV}$ per nucleon but also at other bombarding energies up to 1.5 GeV per nucleon [29].

The Kraków Triple Telescope Array KraTTA [127] was used to improve the capabilities for measuring charged particle flows under the same conditions. With its possibility to identify the masses of light fragments up to beryllium, the study of isospin effects can be extended to the emission properties of the isobar pairs ${ }^{3} \mathrm{H} /{ }^{3} \mathrm{He},{ }^{6} \mathrm{He} /{ }^{6} \mathrm{Li}$, and ${ }^{7} \mathrm{Li} /{ }^{7} \mathrm{Be}$. Yield ratios have been suggested as useful probes for disentangling ambiguities between the effects of the symmetry energy and of the neutron-proton effectivemass splitting in the nuclear medium $[8,32,69]$.

KraTTA had been specifically designed for the experiment to measure the energy, emission angles and isotopic composition of light charged reaction products [127]. Its 35 individual triple telescopes were arranged in a $7 \times 5$ array and placed opposite to LAND on the other side of the beam axis at a distance of $40 \mathrm{~cm}$ from the target. Together, they covered $160 \mathrm{msr}$ of solid angle at polar angles between $\theta_{\text {lab }}=20^{\circ}$ and $64^{\circ}$.

Each KraTTA module consists of two optically decoupled $\mathrm{CsI}(\mathrm{Tl})$ crystals with thicknesses of $2.5 \mathrm{~cm}$ and $12.5 \mathrm{~cm}$ and three large area $500 \mu \mathrm{m}$ thick PIN photodiodes. The first photo-diode serves as a $\Delta E-E$ detector and supplies only an ionization signal. It is followed by the second diode mounted at the front entrance of the short $\mathrm{CsI}(\mathrm{Tl})$ crystal in a single-chip-telescope configuration. It provides both, the intrinsic ionization signal and the light signal provided by the crystal. The third photodiode mounted at the back end of the long crystal provides the light signal from this third element of the configuration. The signals from the photodiodes were processed with custom-made low-noise preamplifiers and digitized with wave form digitizers at a rate of $100 \mathrm{MHz}$. The separation of the ionization and light signals from the second diode and the decomposition of the fast and slow scintillation components were obtained by individually fitting each of the recorded signals [127].

A schematic view of the experimental set-up is shown in fig. 19. The beam was guided in vacuum to about $2 \mathrm{~m}$ upstream from the target. A thin plastic foil read by two photo-multipliers was used to record the projectile arrival times and to serve as a start detector for the time-of-flight 


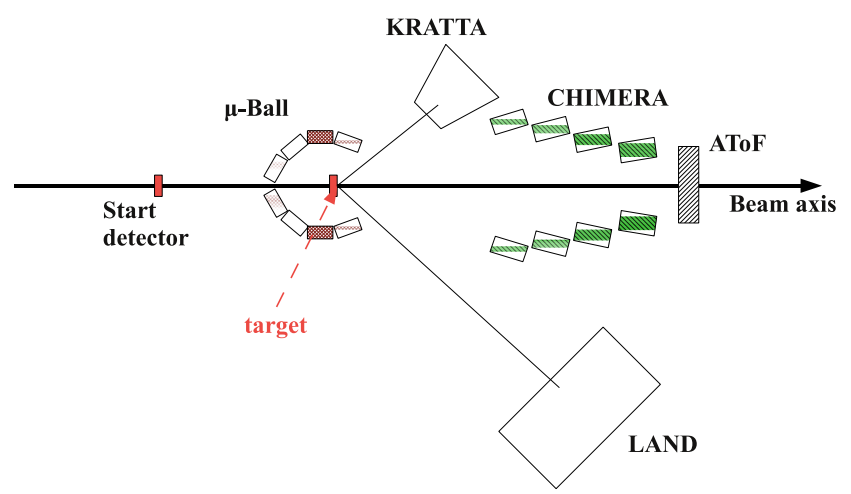

Fig. 19. Schematic view of the experimental setup used in the ASY-EOS experiment S394 at GSI showing the six main detector systems and their positions relative to the beam direction. The dimensions of the symbol and the distances are not to scale (from ref. [128]).

measurement. The Large Area Neutron Detector (LAND, ref. [43]), recently upgraded with new TACQUILA GSIASIC electronics, was positioned at a laboratory angle near $45^{\circ}$ with respect to the beam direction, at a distance of about $5 \mathrm{~m}$ from the target. Its kinematical acceptance was similar to that of the forward LAND subdetector used in the FOPI-LAND experiment (cf. fig. 15) but slightly larger in rapidity for given transverse momentum due to the shorter distance from the target. Also the efficiency was larger because of the full $1 \mathrm{~m}$ depth that has been used (fig. 19). The veto-wall of plastic scintillators in front of LAND permitted the distinction between neutral and charged particles.

The determination of the impact parameter and the orientation of the reaction plane required several detection devices:

i) The ALADIN Time-of-Flight wall [129] was used to detect charged particles and fragments in forward direction at polar angles up to $\theta_{\mathrm{lab}} \leq 7^{\circ}$. The two walls (front and rear) of about $1 \mathrm{~m}^{2}$ active area consisted of vertical arrays of plastic scintillator slabs, each $2.5 \mathrm{~cm}$ wide, $100 \mathrm{~cm}$ long and $1 \mathrm{~cm}$ thick, read out by two photo-multipliers at the upper and lower ends of the slabs. The front and rear walls were displaced by one half of the slab width to gain in horizontal position information. Vertically, a position resolution of about $10 \mathrm{~cm}$ was obtained. Fragment atomic numbers were individually resolved up to about $Z=10$.

ii) 50 thin (between $3.6 \mathrm{~mm}$ and $5.6 \mathrm{~mm}) \mathrm{CsI}(\mathrm{Tl})$ elements of the Washington-University $\mu$-ball array [130], read out by photo-diodes and arranged in 4 rings to cover polar angles between $60^{\circ}$ and $147^{\circ}$. The $\mu$-ball elements surrounded the target with the aim of measuring the azimuthal distribution of particles emitted in backward directions in the c.m. system and to discriminate against background reactions on non-target material;

iii) $352 \mathrm{CsI}(\mathrm{Tl})$ scintillators, $12 \mathrm{~cm}$ thick, of the CHIMERA multidetector [61], arranged in 8 rings in $2 \pi$ azimuthal coverage around the beam axis, covered polar angles between $7^{\circ}$ deg and $20^{\circ}$, measuring the emission of light charged particles. In addition, thin $(300 \mu \mathrm{m})$ Silicon

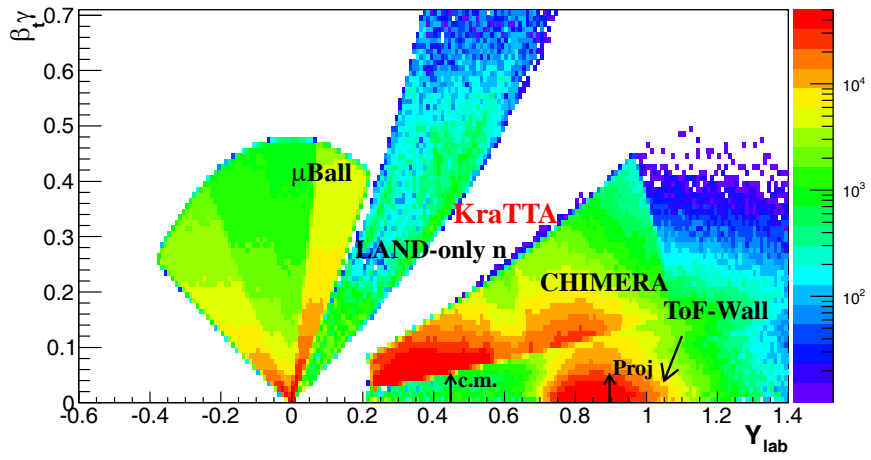

Fig. 20. Invariant hit distribution in the $\gamma \beta_{t} v s$. rapidity $y_{\text {lab }}$ plane for charged particles detected with the three systems $\mu$-Ball, CHIMERA, and ToF-Wall with full azimuthal coverage and neutrons detected with LAND. The acceptance of KRATTA is similar to that of LAND (from ref. [128]).

detectors were placed in front of 32 (4 by ring) CsI detectors to serve as $\Delta E$ detectors. They were used to aid in the analysis of the observed pulse shapes of particles stopped in or punching through the $\mathrm{CsI}(\mathrm{Tl})$ crystals. Particle identification with the CHIMERA elements has been performed using pulse-shape analysis based on standard fast-slow techniques. Isotopic identification is achieved for $p, d, t$, and ${ }^{3,4} \mathrm{He}$ ions stopped in the CsI detectors.

With beam intensities of about $10^{5}$ particles per second and targets of 1-2\% interaction probability, about $5 \cdot 10^{6}$ events for each system were collected. Isotopically enriched metallic ${ }^{96} \mathrm{Ru}$ and oxide ${ }^{96} \mathrm{ZrO}_{2}$ targets with isotopic purities of $96.5 \%$ and $91.4 \%$, respecticely, were used. Special runs were performed with and without target, in order to measure the background from interaction of projectile ions with air, and with iron shadow bars covering the angular acceptance of LAND in order to measure the background of scattered neutrons. The analysis of the collected data is currently still in progress and only preliminary results illustrating the overall system performance are reported here.

As a global result, the coverage of the various detection systems in the transverse velocity vs. rapidity plane is shown in fig. 20 for the ${ }^{197} \mathrm{Au}+{ }^{197} \mathrm{Au}$ system at $400 \mathrm{MeV}$ per nucleon. In the case of the $\mu$-ball, without the necessary information on energy and particle identity, a uniform kinetic-energy distribution from 0 to $100 \mathrm{MeV}$ has been assumed. The populations of two intense regions around mid-rapidity and around the projectile rapidity $y_{p}=0.896$ are clearly discerned. Corrections for acceptance or efficiency have not been applied which, e.g., causes the discontinuity between the projectile sources as seen with CHIMERA and with the ToF-Wall. With common detection thresholds in atomic number $Z$, the discontinuity disappears.

As an example of neutron flow, the azimuthal distributions of neutrons around midrapidity $\left(0.45 \leq y / y_{p} \leq\right.$ 0.55 ), extracted form the first plane of LAND, with respect to the reaction plane orientation given by CHIMERA alone for ${ }^{197} \mathrm{Au}+{ }^{197} \mathrm{Au}$ collisions at $400 \mathrm{MeV}$ per nucleon are shown in fig. 21. The lines represent the results of 


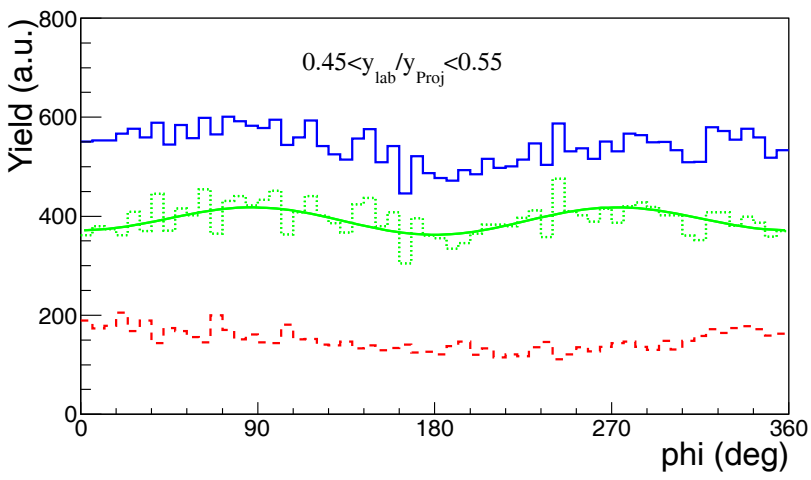

Fig. 21. Azimuthal distributions of neutrons around midrapidity $\left(0.45 \leq y / y_{p} \leq 0.55\right)$, extracted form the first plane of LAND, with respect to the reaction plane orientation given by CHIMERA for ${ }^{197} \mathrm{Au}+{ }^{197} \mathrm{Au}$ collisions at $400 \mathrm{MeV}$ per nucleon. The lines represent the results of measurements without (top histogram, blue) and with (bottom histogram, dashed red) the shadow bar inserted. Their subtraction yields the resulting background-corrected distribution given by the dotted green histogram. The full green line represents a fit according to eq. (3) (from ref. [128]).

measurements without and with the shadow bar inserted. The background intensity is considerable but lacks the squeeze-out pattern seen in the full and in the backgroundcorrected distributions, the latter being obtained by subtraction. A fit according to eq. (3) and correction for the attenuation due to the reaction plane dispersion leads to a preliminary flow parameter $v_{2}=-0.10 \pm 0.01$ for this particular bin in rapidity, integrated over impact parameter and over the transverse-momentum range covered at this rapidity bin (cf. fig. 15). It is of the expected magnitude (fig. 16) but interpretations will have to wait for additional progress of the analysis.

\section{Towards model invariance}

The parameter dependence of the model predictions for the neutron-proton elliptic-flow difference (npEFD) and ratio (npEFR) has recently been investigated in detail [58]. The effects of the selected microscopic nucleon-nucleon cross-sections, of the compressibility of nuclear matter, of the optical potential, and of the parameterization of the symmetry energy were thoroughly studied. The parameterization of the symmetry energy derived from the momentum-dependent Gogny force and a power law parameterization, as presented in sect. 5.2, were both used in conjunction with the Tübingen QMD model and the results were compared with the experimental FOPI/LAND data for ${ }^{197} \mathrm{Au}+{ }^{197} \mathrm{Au}$ collisions at $400 \mathrm{MeV}$ per nucleon. The acceptance cuts of the FOPI/LAND experiment were applied. It was found that the global variation of the general model parameters within commonly accepted limits leads to finite uncertainties of typically one unit of the $x$ parameter describing the stiffness of the symmetry energy in the MDI parameterization $[7,53,54]$.

The predictions for the npEFD and npEFR are displayed in figs. 22 and 23, respectively, as a function of the

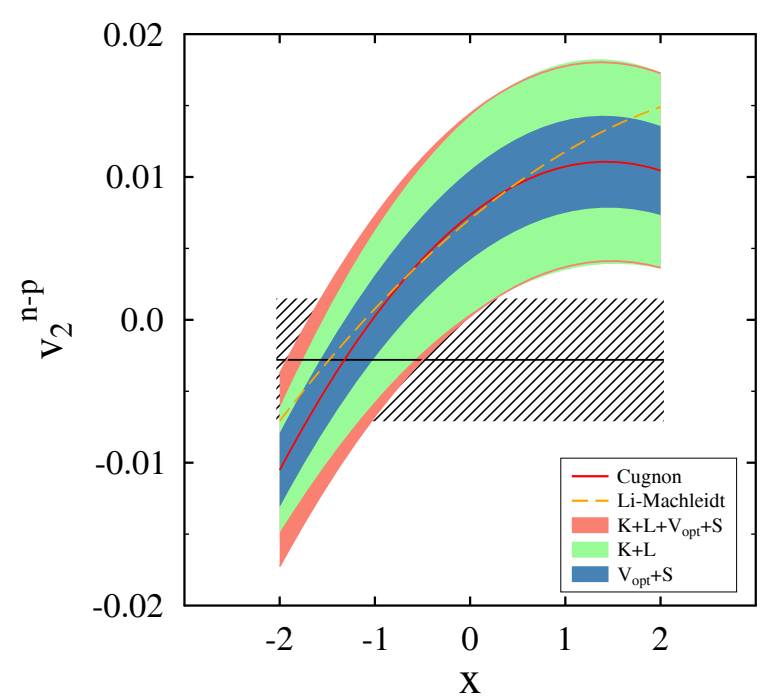

Fig. 22. Model dependence of npEFD in comparison with FOPI-LAND experimental data, integrated over impact parameter $b<7.5 \mathrm{fm}$, normalized rapidity $0.25<y / y_{p}<0.75$, and transverse momentum $0.3<p_{t}<1.0 \mathrm{GeV} / c$. The sensitivities to the model parameters compressibility modulus $(K)$, width of the nucleon wave function $(L)$, optical potential $\left(V_{\text {opt }}\right)$, and parameterization of the symmetry energy $(S)$ are displayed. The total model dependence is obtained by adding in quadrature individual sensitivities.

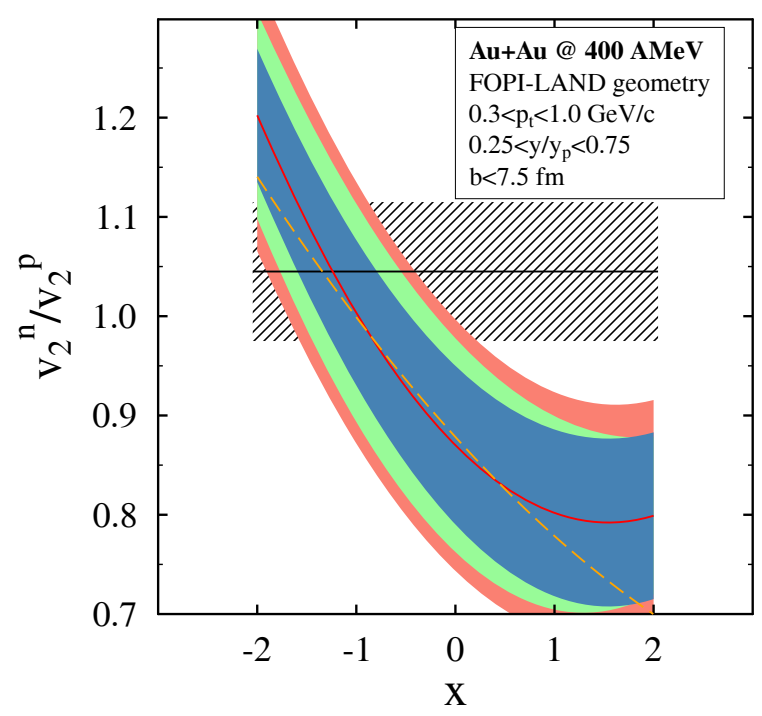

Fig. 23. The same as fig. 22 but for npEFR.

stiffness of the asy-EoS. The sensitivity to all model parameters studied in refs. $[40,58]$ and a comparison to the latest impact parameter integrated FOPI/LAND data are included. As in ref. [58], the central theoretical results were obtained with a set of model parameters that best reproduce the experimental values for $v_{2}^{n}$ and $v_{2}^{p}$ but, in contrast to there, the variations of $K$ and $L$ were not subject to the constraint that the elliptic flow values stay within a limit of $25 \%$ with respect to the experimental results. Effectively, this has only consequences for the sensitivity to the compressibility modulus which has been varied within 
the range $K=190-300 \mathrm{MeV}$, giving rise to departures of $v_{2}$ from the experimental values by as much as $40-50 \%$.

The sensitivities due to the optical potential and symmetry energy parametrizations represent averages over the three and, respectively, two scenarios that were employed here as well as in ref. [58] for the two model ingredients. The results shown in figs. 22 and 23 have been obtained by adding in quadrature these two uncertainties. Conclusions as, e.g., regarding the sensitivity/insensitivity of the studied observables to the momentum dependence of the symmetry energy (as was presented for an asy-soft scenario in ref. [131]) cannot be directly deduced from these figures. Each of the possible combinations of the parameterizations of the optical potential and of the symmetry energy usually yields a different outcome in this respect. Nevertheless, it can be concluded that the uncertainties in the optical potential and the momentum dependence/independence of the symmetry energy have an important impact on elliptic flow observables like npEFD and npEFR. A precise constraining of the high-density symmetry energy dependence from elliptic flow data will, therefore, require an accurate knowledge of the optical potential and the resolution of the problem concerning the momentum dependence/independence of asy-EoS.

The hatched bands shown in figs. 22 and 23 represent the experimental results obtained from the reanalysis of the FOPI-LAND experiment. Their errors of mainly statistical nature contribute an additional uncertainty, comparable to the overall parameter dependence. Even with more precise data, a considerable uncertainty related to the dependence on model parameters will still remain. It is to be expected, however, that the model dependence can be further reduced by explicitly studying trends of the measured observables as a function of impact parameter, transverse momentum, and rapidity. At present, altogether, a moderately stiff, $x=-1.35 \pm 1.25$, symmetry energy was extracted from this study, a result that is slightly stiffer but compatible with that of the similar study with the UrQMD transport model and a momentum-independent power law parameterization of the symmetry energy.

The uncertainty of $x$ is of statistical nature as far as it originates from the experimental errors but mainly systematic regarding the predictions. The widths of the theoretical error bands are the result of the different scenarios that were considered acceptable. Imposing stricter constraints will result in narrower bands. The theoretical errors, statistical as well as numerical, are about 0.040 for npEFD and 0.035 for npEFR in absolute values, i.e. about half of the widths of the bands. In the super-soft region, the separation between the theoretical and experimental central values is thus of the order of three sigma.

In fig. 24, the explicit constraints on the density dependence of the symmetry energy obtained in this study from the comparison of theoretical and experimental values of $n p E F D$ and npEFR are presented. For comparison, the result of ref. [39] is added. The two studies employ independent flavors of the QMD transport model (Tübingen QMD vs. UrQMD) and parameterizations of both, the isoscalar and isovector EoS that differ: Gogny inspired

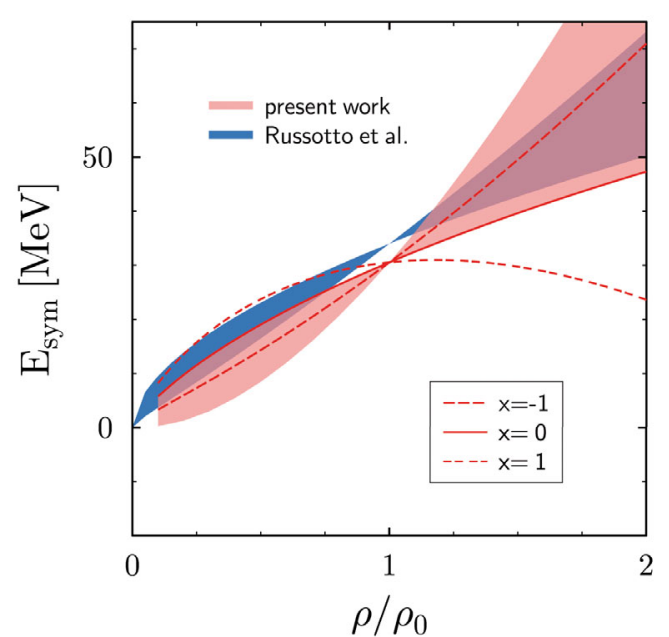

Fig. 24. Constraints on the density dependence of symmetry energy obtained from comparing theoretical predictions for npEFD and npEFR to FOPI-LAND experimental data. The result of ref. [39] is also shown together with the Gogny inspired parameterization of the symmetry energy for three values of the stiffness parameter: $x=-1$ (stiff), $x=0$ and $x=1$ (soft) (reprinted with permission from ref. [58]; Copyright (2013) by the American Physical Society).

vs. Hartnack-Aichelin parameterization [121] of the optical potential (that also differ in their energy dependence) and Gogny-inspired (momentum dependent) vs. power law parameterization (momentum independent) asy-EoS. The constraints on the density dependence of the symmetry energy obtained with these different ingredients are in agreement with each other which contrasts with the current status of the effort to constrain the symmetry energy from $\pi^{-} / \pi^{+}$ratios. By combining the two estimates, a moderately stiff to linear density dependence corresponding to a parameterization $x=-1.0 \pm 1.0$ is obtained. It indicates a somewhat faster increase of the symmetry energy with density than what is extracted from nuclear structure and reactions for sub-saturation densities.

\section{Conclusion and outlook}

According to the predictions of transport models, the relative strengths of neutron and proton elliptic flows represent an observable sensitive to the symmetry energy at densities near and above saturation. By forming ratios or differences of neutron $v s$. proton or neutron $v s$. hydrogen flows, the influence of isoscalar-type parameters of the model descriptions can be minimized.

The performed comparisons of the existing results of the FOPI/LAND experiment for ${ }^{197} \mathrm{Au}+{ }^{197} \mathrm{Au}$ reactions at $400 \mathrm{MeV}$ per nucleon with UrQMD transport calculations favor a moderately soft to linear symmetry term with a density dependence of the potential term proportional to $\left(\rho / \rho_{0}\right)^{\gamma}$ with $\gamma=0.9 \pm 0.4$, compatible with predictions of ab initio calculations. A similar result was obtained by studying the neutron-proton flow differences from the same experiment with the Tübingen QMD 
model. Combining the estimates of both studies leads to the following constraint, obtained from averaging these results. Expressed in terms of the MDI parameterization, the stiffness of the symmetry energy as a function of density is given by $x=-1.0 \pm 1.0$. It corresponds to a moderately stiff to linear density dependence and excludes the super-soft and, with a lesser degree of confidence, the soft asy-EoS scenarios from the list of possibilities.

Collective flows have proven to be useful probes of nuclear matter properties at high density. However, the explicit proof that the elliptic-flow ratios are probing the isovector component of the nuclear mean field at suprasaturation densities is, so far, limited to test calculations made with the UrQMD. They indicate that the strength of the symmetry energy at densities both, below and above saturation, are essential. It will be useful to study this in more detail.

The statistical uncertainty of the existing FOPI/ LAND data is larger than the investigated systematic effects of model parameters and analysis techniques. A significantly improved result can thus be expected from a new experiment delivering a comprehensive data set with sufficiently high statistical accuracy. Explicitly testing the predicted dependences on rapidity and transverse momentum will provide useful constraints for the models. Additional observables as, e.g., neutron-to-proton or ${ }^{3} \mathrm{H}$-to- ${ }^{3} \mathrm{He}$ yield ratios may serve in resolving ambiguities caused by the effective-mass splitting between neutrons and protons. It will be interesting to see first results appearing from the recently completed measurements of the ASY-EOS Collaboration at the GSI laboratory.

The parameter dependence of the predictions for the differential observables flow ratio and flow difference by transport models has been systematically studied with the Tübingen QMD model [58]. It has been concluded that, while the sensitivity to uncertainties in the model parameters is important, the two observables offer the opportunity to extract information about the symmetry energy above the saturation point. Furthermore, the results of this study supplemented with those of Russotto et al. [39] allow one to conclude that constraints for the symmetry energy extracted from elliptic flow data are independent of its parameterization, suggesting that an almost modelindependent extraction can be achieved in this case. This contrasts with the case of $\pi^{-} / \pi^{+}$ratios where the stiffnesses of asy-EoS extracted with different parameterizations or transport models can be extremely different.

New theoretical developments as well as new experimental measurements of meson spectra, together with the exploitation of existing data on meson directed and elliptic flows [47], may prove of great importance for approaching a resolution of the currently existing pion-ratio problem. It will represent a crucial test of our proper understanding of hadronic interactions and their in-medium counterparts in the energy regime around and below $1 \mathrm{GeV}$ per nucleon. Tighter constraints on the isovector part of the equation of state from elliptic flow of nucleons and light clusters appear to be desirable and attainable, motivating present and future efforts in this direction, both experimentally and theoretically.
Because of the quadratically rising importance of the symmetry energy, the continuation of this program with systems of larger asymmetry is very promising and important. The lower luminosities to be expected from the use of secondary beams and isotopically enriched targets will have to be compensated with efficient detector setups. The neutron detector NeuLAND proposed for experiments at FAIR will offer a highly improved detection efficiency for neutrons in the energy range 100 to $400 \mathrm{MeV}$ [132]. This will be essential for extending the program also to reactions at lower energies for which significant mean-field effects are predicted for directed and elliptic flows [55].

Stimulating and fruitful discussions with W. Reisdorf, H.H. Wolter, and many colleagues within the ASY-EOS Collaboration (ref. [60]) are gratefully acknowledged.

Open Access This is an open access article distributed under the terms of the Creative Commons Attribution License (http://creativecommons.org/licenses/by/4.0), which permits unrestricted use, distribution, and reproduction in any medium, provided the original work is properly cited.

\section{References}

1. STAR Collaboration (B.I. Abelev et al.), Phys. Rev. Lett. 99, 112301 (2007).

2. ALICE Collaboration (K. Aamodt et al.), Phys. Rev. Lett. 105, 252302 (2010).

3. PHENIX Collaboration (A. Adare et al.), Phys. Rev. C 85, 064914 (2012).

4. ATLAS Collaboration (G. Aad et al.), Phys. Lett. B 707, 330 (2012).

5. Jun Xu, Lie-Wen Chen, Che Ming Ko, Bao-An Li, YuGang Ma, Phys. Rev. C 87, 067601 (2013).

6. J.M. Lattimer, M. Prakash, Phys. Rep. 442, 109 (2007).

7. Bao-An Li, Lie-Wen Chen, Che Ming Ko, Phys. Rep. 464, 113 (2008).

8. M. Di Toro, V. Baran, M. Colonna, V. Greco, J. Phys. G 37, 083101 (2010).

9. P.B. Demorest, T. Pennucci, S.M. Ransom, M.S.E. Roberts, J.W.T. Hessels, Nature 467, 1081 (2010).

10. C. Fuchs, H.H. Wolter, Eur. Phys. J. A 30, 5 (2006).

11. P. Danielewicz, Nucl. Phys. A 673, 375 (2000).

12. P. Danielewicz, R. Lacey, W.G. Lynch, Science 298, 1592 (2002).

13. C. Sturm et al., Phys. Rev. Lett. 86, 39 (2001).

14. C. Fuchs, A. Faessler, E. Zabrodin, Y.M. Zheng, Phys. Rev. Lett. 86, 1974 (2001).

15. Ch. Hartnack, H. Oeschler, J. Aichelin, Phys. Rev. Lett. 96, 012302 (2006).

16. M.B. Tsang et al., Phys. Rev. C 86, 015803 (2012).

17. P. Möller, W.D. Myers, H. Sagawa, S. Yoshida, Phys. Rev. Lett. 108, 052501 (2012).

18. Lie-Wen Chen, arXiv:1212.0284 [nucl-th] (2012).

19. B.A. Brown, Phys. Rev. Lett. 85, 5296 (2000).

20. R. Subedi et al., Science 320, 1476 (2008).

21. Chang Xu, Bao-An Li,, Phys. Rev. C 81, 064612 (2010).

22. A.W. Steiner, S. Gandolfi, Phys. Rev. Lett. 108, 081102 (2012).

23. K. Hebeler, A. Schwenk, Phys. Rev. C 82, 014314 (2010). 
24. A. Carbone, A. Polls, A. Rios, EPL 97, 22001 (2012).

25. M. Alvioli, C. Ciofi degli Atti, L.P. Kaptari, C.B. Mezzetti, H. Morita, Int. J. Mod. Phys. E 22, 1330021 (2013).

26. W. Reisdorf, H.G. Ritter, Annu. Rev. Nucl. Part. Sci. 47, 663 (1997).

27. N. Herrmann, J.P. Wessels, T. Wienold, Annu. Rev. Nucl. Part. Sci. 49, 581 (1999)

28. H. Stöcker, W. Greiner, Phys. Rep. 137, 277 (1986).

29. W. Reisdorf et al., Nucl. Phys. A 876, 1 (2012).

30. Bao-An Li, Phys. Rev. Lett. 88, 192701 (2002).

31. Q. Li, Z. Li, H. Stöcker, Phys. Rev. C 73, 051601 (2006).

32. Zhao-Qing Feng, Phys. Lett. B 707, 83 (2012).

33. M. Famiano et al., Phys. Rev. Lett. 97, 052701 (2006).

34. L. Scalone, M. Colonna, M. Di Toro, Phys. Lett. B 461, 9 (1999).

35. Bao-An Li, A.T. Sustich, Bin Zhang, Phys. Rev. C 64, 054604 (2001).

36. V. Greco, V. Baran, M. Colonna, M. Di Toro, T. Gaitanos, H.H. Wolter, Phys. Lett. B 562, 215 (2003).

37. V. Baran, M. Colonna, V. Greco, M. Di Toro, Phys. Rep. 410, 335 (2005).

38. H.H. Gutbrod, K.H. Kampert, B. Kolb, A.M. Poskanzer, H.G. Ritter, R. Schicker, H.R. Schmidt, Phys. Rev. C 42, 640 (1990).

39. P. Russotto et al., Phys. Lett. B 697, 471 (2011).

40. M.D. Cozma, Phys. Lett. B 700, 139 (2011).

41. Y. Leifels et al., Phys. Rev. Lett. 71, 963 (1993).

42. D. Lambrecht et al., Z. Phys. A 350, 115 (1994).

43. Th. Blaich et al., Nucl. Instrum. Methods Phys. Res. A 314, 136 (1992).

44. Q. Li, Z. Li, S. Soff, R.K. Gupta, M. Bleicher, H. Stöcker, J. Phys. G 31, 1359 (2005).

45. G. Ferini, T. Gaitanos, M. Colonna, M. Di Toro, H.H. Wolter, Phys. Rev. Lett. 97, 202301 (2006).

46. X. Lopez et al., Phys. Rev. C 75, 011901(R) (2007).

47. W. Reisdorf et al., Nucl. Phys. A 781, 459 (2007).

48. Zhigang Xiao et al., Phys. Rev. Lett. 102, 062502 (2009).

49. Zhao-Qing Feng, Gen-Ming Jin, Phys. Lett. B 683, 140 (2010).

50. W.-J. Xie, J. Su, L. Zhu, F.-S. Zhang, Phys. Lett. B 718, 1510 (2013).

51. De-Hua Wen, Bao-An Li, Lie-Wen Chen, Phys. Rev. Lett. 103, 211102 (2009)

52. Bao-An Li et al., J. Phys. Conf. Ser. 312, 042006 (2011).

53. C.B. Das, S. Das Gupta, C. Gale, Bao-An Li, Phys. Rev. C 67, 034611 (2003).

54. Bao-An Li, Lie-Wen Chen, Phys. Rev. C 72, 064611 (2005).

55. ChenChen Guo, YongJia Wang, QingFeng Li, W. Trautmann, Ling Liu, LiJuan Wu, Sci. China Phys. Mech. Astron. 55, 252 (2012).

56. G. Ferini, M. Colonna, T. Gaitanos, M. Di Toro, Nucl. Phys. A 762, 147 (2005).

57. Hermann Wolter, Proceedings of Science (Bormio2012), 059 (2012).

58. M.D. Cozma, Y. Leifels, W. Trautmann, Q. Li, P. Russotto, Phys. Rev. C 88, 044912 (2013).

59. R.C. Lemmon et al., proposal for SIS experiment S394 (2009).

60. P. Russotto et al., J. Phys. Conf. Ser. 420, 012092 (2013) arXiv:1209.5961 [nucl-ex].

61. A. Pagano et al., Nucl. Phys. A 734, 504 (2004).
62. W. Trautmann, H.H. Wolter, Int. J. Mod. Phys. E 21, 1230003 (2012).

63. W.D. Myers, W.J. Swiatecki, Nucl. Phys. 81, 1 (1966).

64. M. Baldo, C. Maieron, P. Schuck, X. Viñas, Nucl. Phys. A 736, 241 (2004).

65. R.B. Wiringa, S.C. Pieper, Phys. Rev. Lett. 89, 182501 (2002).

66. Z.H. Li, U. Lombardo, H.-J. Schulze, W. Zuo, L.W. Chen, H.R. Ma, Phys. Rev. C 74, 047304 (2006).

67. F. Burgio, J. Phys. G 35, 014048 (2008).

68. K. Hebeler, J.M. Lattimer, C.J. Pethick, A. Schwenk, Phys. Rev. Lett. 105, 161102 (2010).

69. V. Giordano, M. Colonna, M. Di Toro, V. Greco, J. Rizzo, Phys. Rev. C 81, 044611 (2010).

70. Q. Li, Z. Li, S. Soff, M. Bleicher, H. Stöcker, J. Phys. G 32, 151 (2006).

71. Q. Li, Z. Li, S. Soff, M. Bleicher, H. Stöcker, J. Phys. G 32, 407 (2006).

72. D.T. Khoa, N. Ohtsuka, M.A. Matin, A. Faessler, S.W. Huang, E. Lehmann, R.K. Puri, Nucl. Phys. A 548, 102 (1992).

73. V.S. Uma Maheswari, C. Fuchs, A. Faessler, L. Sehn, D.S. Kosov, Z. Wang, Nucl. Phys. A 628, 669 (1998).

74. M.B. Tsang et al., Phys. Rev. Lett. 102, 122701 (2009).

75. J.M. Lattimer, Y. Lim, Astrophys. J. 771, 51 (2013).

76. A.W. Steiner, J.M. Lattimer, E.F. Brown, Astrophys. J. $\mathbf{7 2 2}, 33$ (2010).

77. A.W. Steiner, J.M. Lattimer, E.F. Brown, Astrophys. J. 765, L5 (2013).

78. Bao-An Li, Lie-Wen Chen, F.J. Fattoyev, W.G. Newton, Chang Xu, arXiv:1212.1178 [nucl-th] (2012).

79. I. Vidaña, C. Providência, A. Polls, A. Rios, Phys. Rev. C 80, 045806 (2009).

80. S. Abrahamyan et al., Phys. Rev. Lett. 108, 112502 (2012).

81. C.J. Horowitz et al., Phys. Rev. C 85, 032501(R) (2012).

82. G.F. Bertsch, W.G. Lynch, M.B. Tsang, Phys. Lett. B 189, 384 (1987)

83. R.C. Lemmon et al., Phys. Lett. B 446, 197 (1999).

84. G.D. Westfall, Nucl. Phys. A 630, 27c (1998).

85. G.D. Westfall et al., Phys. Rev. Lett. 71, 1986 (1993).

86. R. Pak et al., Phys. Rev. Lett. 78, 1022 (1997).

87. R. Pak et al., Phys. Rev. Lett. 78, 1026 (1997).

88. Yingxun Zhang, Zhuxia Li, Phys. Rev. C 74, 014602 (2006).

89. Z. Kohley et al., Phys. Rev. C 82, 064601 (2010).

90. Z. Kohley et al., Phys. Rev. C 85, 064605 (2012).

91. Qingfeng Li, C. Shen, C. Guo, Y. Wang, Z. Li, J. Lukasik, W. Trautmann, Phys. Rev. C 83, 044617 (2011).

92. Bao-An Li, Nucl. Phys. A 708, 365 (2002).

93. Gao-Chan Yong, Bao-An Li, Lie-Wen Chen, Phys. Rev. C 74, 064617 (2006).

94. S. Voloshin, Y. Zhang, Z. Phys. C 70, 665 (1996).

95. J.-Y. Ollitrault, arXiv:nucl-ex/9711003 (1997).

96. A.M. Poskanzer, S.A. Voloshin, Phys. Rev. C 58, 1671 (1998).

97. A. Andronic et al., Phys. Rev. C 64, 041604 (2001).

98. A. Andronic, J. Łukasik, W. Reisdorf, W. Trautmann, Eur. Phys. J. A 30, 31 (2006).

99. J. Łukasik et al., Phys. Lett. B 608, 223 (2005).

100. A. Andronic et al., Phys. Lett. B 612, 173 (2005).

101. C. Pinkenburg et al., Phys. Rev. Lett. 83, 1295 (1999). 
102. P. Braun-Munzinger, J. Stachel, Nucl. Phys. A 638, 3c (1998).

103. M.B. Tsang et al., Phys. Rev. C 47, 2717 (1993).

104. R. Fries, V. Greco, P. Sorensen, Annu. Rev. Nucl. Part. Sci. 58, 177 (2008).

105. R. Snellings, New J. Phys. 13, 055008 (2011).

106. P. Danielewicz, G. Odyniec, Phys. Lett. B 157, 146 (1985).

107. J. Łukasik, W. Trautmann, in Proceedings of the IWM2005 International Workshop on Multifragmentation and Related Topics, Catania, Italy, 2005, edited by R. Bougault et al., SIF Conf. Proc., Vol. 91 (Italian Physical Society, Bologna, 2006) p. 387, arXiv:nuclex/0603028 (2006).

108. J. Łukasik, W. Trautmann, arXiv:0708.2821 [nucl-ex] (2007).

109. C. Hartnack, Li. Zhuxia, L. Neise, G. Peilert, A. Rosenhauer, H. Sorge, J. Aichelin, H. Stöcker, W. Greiner, Nucl. Phys. A 495, 303c (1989).

110. S.A. Bass et al., Progr. Part. Nucl. Phys. 41, 225 (1998).

111. Q. Li, M. Bleicher, J. Phys. G 36, 015111 (2009).

112. Qingfeng Li, Caiwan Shen, M. Di Toro, Mod. Phys. Lett. A 25, 669 (2010).

113. G.Q. Li, R. Machleidt, Phys. Rev. C 48, 1702 (1993).

114. G.Q. Li, R. Machleidt, Phys. Rev. C 49, 566 (1994).

115. C. Fuchs, A. Faessler, M. El-Shabshiry, Phys. Rev. C 64, 024003 (2001).

116. Q. Li, Z. Li, E. Zhao, Phys. Rev. C 69, 017601 (2004).

117. Y. Wang, C. Guo, Q. Li, H. Zhang, Z. Li, W. Trautmann, arXiv:1305.4730 [nucl-th] (2013).

118. Q. Li, Z. Li, E. Zhao, R.K. Gupta, Phys. Rev. C 71 , 054907 (2005).
119. Y. Wang, C. Guo, Q. Li, H. Zhang, Sci. China Phys. Mech. Astron. 55, 2407 (2012).

120. M. Baldo, I. Bombaci, G. Giansiracusa, U. Lombardo, Phys. Rev. C 40, R491 (1989).

121. C. Hartnack, J. Aichelin, Phys. Rev. C 49, 2801 (1994).

122. Ch. Hartnack, R.K. Puri, J. Aichelin, J. Konopka, S.A. Bass, H. Stöcker, W. Greiner, Eur. Phys. J. A 1, 151 (1998) the notation for $L$ follows the convention used in this reference.

123. K. Shekhter, C. Fuchs, A. Faessler, M. Krivoruchenko, B. Martemyanov, Phys. Rev. C 68, 014904 (2003).

124. E. Santini, M.D. Cozma, A. Faessler, C. Fuchs, M.I. Krivoruchenko, B. Martemyanov, Phys. Rev. C 78, 034910 (2008).

125. M.D. Cozma, C. Fuchs, E. Santini, A. Fassler, Phys. Lett. B 640, 170 (2006).

126. C. Fuchs, P. Essler, T. Gaitanos, H.H. Wolter, Nucl. Phys. A 626, 987 (1997).

127. J. Łukasik et al., Nucl. Instrum. Methods Phys. Res. A 709, 120 (2013).

128. P. Russotto et al., in Proceedings of the International Nuclear Physics Conference INPC2013, Firenze, Italy, 2013, to be pulished in EPJ Web of Conferences (2013).

129. A. Schüttauf et al., Nucl. Phys. A 607, 457 (1996).

130. D.G. Sarantites, P.-F. Hua, M. Devlin, L.G. Sobotka, J. Elson, J.T. Hood, D.R. LaFosse, J.E. Sarantites, M.R. Maier, Nucl. Instrum. Methods A 381, 418 (1996).

131. L. Zhang, Y. Gao, Y. Du, G.H. Zuo, G.C. Yong, Eur. Phys. J. A 48, 30 (2012).

132. NeuLAND Technical Design Report, http://fair-center.de/fileadmin/fair/experiments/ NUSTAR/Pdf/TDRs/NeuLAND-TDR-Web.pdf. 